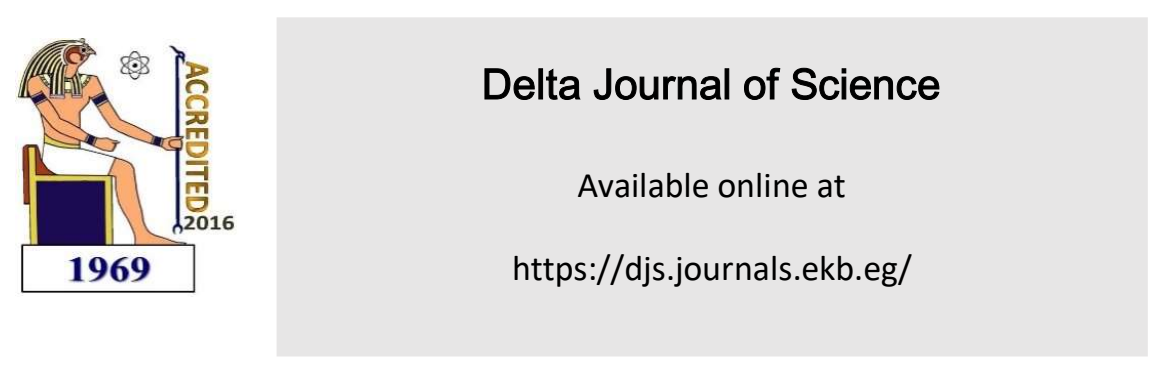

Research Article

MATHEMATICS

\title{
The truncated distribution of the range for a Wiener process: Application to the stock price
}

\author{
Abd El-Moneim A. Teamah ${ }^{1}$, Mohamed A. El-Hadidy ${ }^{2}$ and Maraw M. El-Ghoul ${ }^{3}$ \\ 1,2,3 Department of Mathematics, Faculty of Science, Tanta University, Tanta, Egypt. \\ E-mail addresses: ${ }^{1}$ teamah4@hotmail.com, ${ }^{2}$ melhadidi@science.tanta.edu.eg and \\ 33arwa_mabrouk@science.tanta.edu.eg.
}

Abstract: In this paper, the exact truncated distribution of the stock price (truncated distribution for the range of a Wiener process) is available among the established results in the field of mathematics (Probability Distributions). Various statistical properties of the distribution are derived including reliability properties, moments, stress-strength parameter, order statistics, Bonferroni curve, Lorenz curve and Gini's index. A real data set is analyzed to clarify the effectiveness of this distribution.

Keywords: Truncated distribution; Wiener process; Reliability properties; Order Statistics.

\section{Introduction}

Truncation in probability distributions may occur in many studies such as life testing and reliability. Truncation arises because, in many situations, failure of a unit is observed only if it fails before and/or after a certain period. May sometimes happen to be range of the definition of a certain probabilistic distribution is not fully compatible with some of the data, either for theoretical reasons or because the portion of the data cannot be obtained within this range, in this case we resort to the truncated distribution. The truncation method of the distribution is an important methodology in different fields of sciences, in particular communication networks and finance. etc. Truncation occurs in various situations, for example, right truncation occurs in the study of life testing and reliability of items such as an electronic component, light bulbs, etc. Left truncation arises because, in many situations, failure of a unit is observed only if it fails after a certain period. Often, study units may not be followed at the beginning of an experiment until all of them fail, and the experimenter may have to start at a certain time and stop at a certain time when some of the units may still be working. Many researchers were interested in studying the truncation method of the distribution, for example: Zaninetti [12] 
presents a right and left truncated gamma distribution with application to the stars that introduces an upper and a lower boundary. In addition, the parameters which characterize the truncated gamma distribution are evaluated. A Class of truncated Binomial lifetime distributions is obtained by Alkarni [13]. The type of middle and random truncation have been studied by Mohie ElDin et al. [14] and Teamah et al. [15]. Ali and Nadarajah [3] introduced a truncated version of the Pareto distribution. They derived the explicit expressions for the moments for the truncated version. Nadarajah [4] introduced truncated versions for five of the most commonly known long tailed distributions which possess finite moments of all orders and could therefore be better models. Zaninetti and Ferraro [5] presented a comparison between the Pareto and truncated Pareto distributions. Recently, many papers has been presented the most important applications of the truncated distribution in various fields of science, for example, Pender [7] used the truncated normal distribution to approximate the non stationary single server queue with abandonment. Chattopadhyay et al. [8] provided a more accurate data fitting by using truncated geometric distribution to model the node degree distribution of a network compared to power-law, log-normal, Pareto, drift power-law and power-law with exponential cutoff distributions.

The Wiener process has many applications throughout the mathematical sciences. In physics it is used to study Brownian motion, the diffusion of minute particles suspended in fluid, and other types of diffusion via the Fokker-Planck and Langevin equations. It also forms the basis for the rigorous path integral formulation of quantum mechanics (by the Feynman-Kac formula, a solution to the Schrödinger equation can be represented in terms of the Wiener process) and the study of eternal inflation in physical cosmology. It is also a prominent in the mathematical theory of finance, in particular the Black-Scholes option pricing model. The change of price formula based on the assumption that stock price follow a wiener process. The distribution of stock price through known time interval is the distribution of a Wiener process range. In the time interval $(0, T)$ the range of the Wiener process $\{W(t) ; t \geq 0\}$ is $\bar{R}(T)=\sup _{(0, T)} W(t)-\inf _{(0, T)} W(t)$ and it gives the difference between the highest price for the stock and it's the lowest price. Feller [1] derived the probability density function of this range by using the method of images. Recently, an expansion for its cumulative distribution function and its quantiles are given by Withers and Nadarajah [2]. In addition, they gave a table of this cumulative distribution function. Here we have the following question: what should be done if we need to find the new distribution of the stock price in the time interval $(0, T)$ and its value is sandwiched between two certain values $a, b$ ? To answer the above questions, we should do a truncation on the distribution of a Wiener process range that has been obtained by Feller [1].

In this paper, we will provide the Truncated Distribution of a Wiener Range (TDWR) and study various its statistical properties. The properties studied include reliability properties, moments, stress-strength parameter, order statistics, Bonferroni curve, Lorenz curve and Gini's index. The difference between the TDWR and distribution of a Wiener process range which has been obtained by Feller [1] are showed as in the given figures through the paper.

The paper is organized as follows. In Section 2, we introduce the TDWR. We study some 
statistical properties for TDWR in Section 3. An application to a real data set is presented in Section 4. Section 5 ends the paper with some concluding remarks and future works.

\section{Truncated distribution of a Wiener range (TDWR)}

The stock price is assumed to move randomly according to one dimensional Wiener process $\left\{W(t), t \in \mathrm{R}^{+}\right\}$, where $\mathrm{R}^{+}$is the set of real numbers and $W(t)$ is a Wiener process on $(0, \infty)$ with range $\bar{R}(T)$ on the time interval $(0, T)$. This range is the difference between $\sup _{(0, T)} W(t)$ and $\inf _{(0, T)} W(t)$. Feller [1] gave the probability density function for the range of $W(t)$ which controls the target's motion as:

$$
\begin{aligned}
f_{\bar{R}(T)}(\bar{r})=\left(\frac{2}{\pi}\right)^{\frac{1}{2}} \bar{r}^{-1}(2 \pi)^{\frac{1}{2}}\left(\frac{\bar{r} T^{-\frac{1}{2}}}{2}\right)^{-1} \times \\
\sum_{k=1}^{\infty} \exp \left[-\frac{(2 k-1)^{2} \pi^{2}}{8} \cdot\left(\frac{\bar{r} T^{-\frac{1}{2}}}{2}\right)^{-2}\right]
\end{aligned}
$$

where $0<\bar{r}<\infty$ and $T>0$ and it is represented as in the figure 1 .

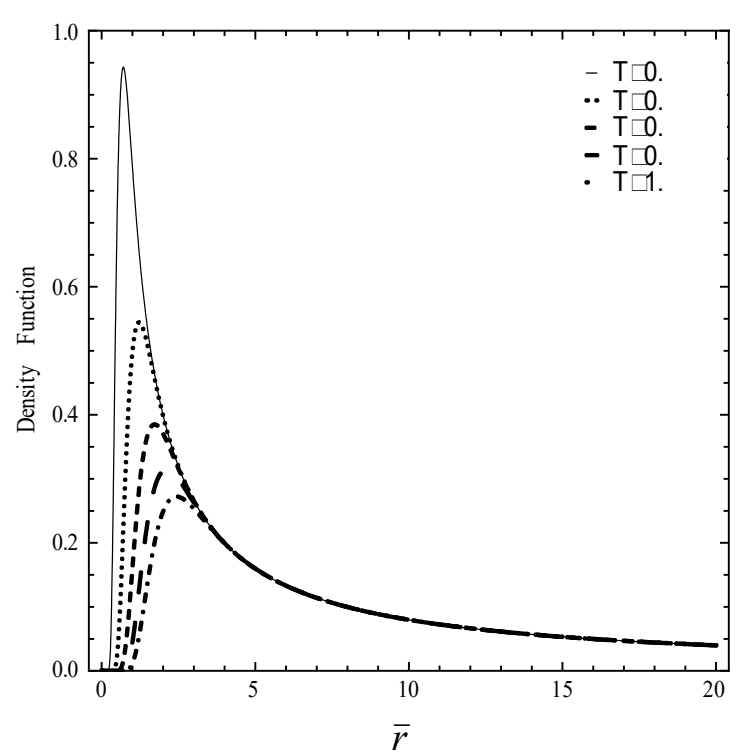

Figure 1: The probability density function of $\bar{R}(T)$.

Withers and Nadarajah [2] give its cumulative distribution function by:

$$
\begin{aligned}
F_{\bar{R}(T)}(\bar{r})=\sum_{k=1}^{\infty}\left(\frac{8}{(2 k-1)^{2} \pi^{2}}+\frac{8 T}{\bar{r}^{2}}\right) \times \\
\exp \left[-\frac{(2 k-1)^{2} \pi^{2} T}{2 \bar{r}^{2}}\right]
\end{aligned}
$$

and it is represented as in figure 2.

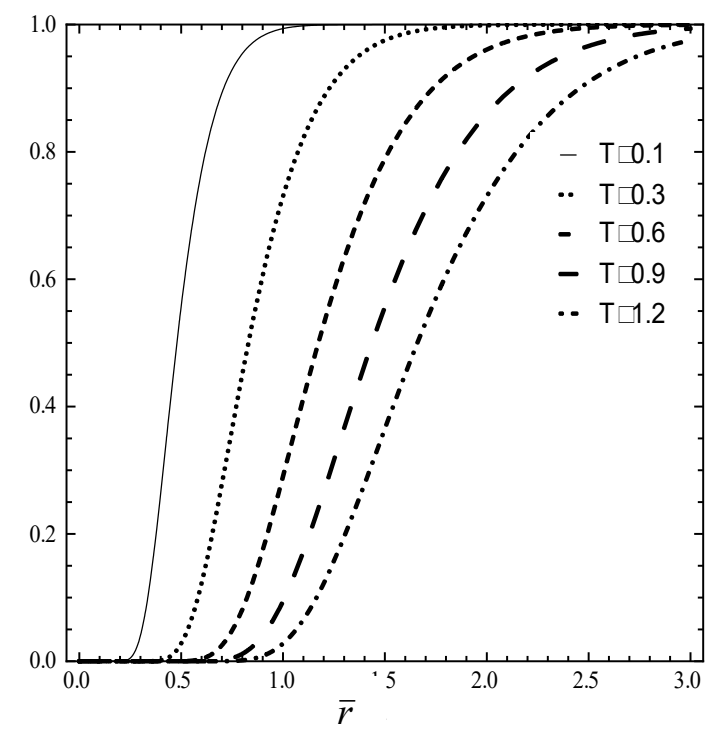

Figure 2: Cumulative distribution function 
Hence, it is easy to show that the survival function $\bar{F}_{\bar{R}(T)}()=.1-F_{\bar{R}(T)}(\bar{r})$ is decreasing by increasing the value of $T$, see figure 3 .

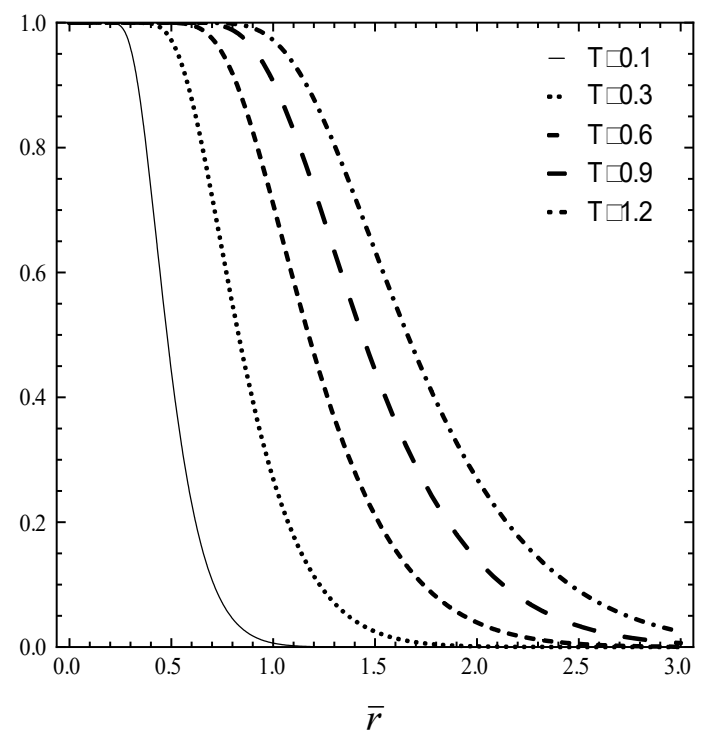

The

Figure 3: Survival function of the range range that uenmed vy (1) hes in lis avinty to model lifetime data with increasing failure rate.

We are interested in TDWR defined by the following definition.

Definition 1. Let $\bar{R}(T)$ be a random variable with probability density function (1), define $R(T)$ as a corresponding double truncated (truncation from left and right) of $\bar{R}(T)$ with the probability density function $g_{R(T)}(r)$ : Then, the probability density function of double truncated of $\bar{R}(T)$ is given by:

$g_{R(T)}(r)=\frac{f_{\bar{R}(T)}(r)}{F_{\bar{R}(T)}(b)-F_{\bar{R}(T)}(a)}$

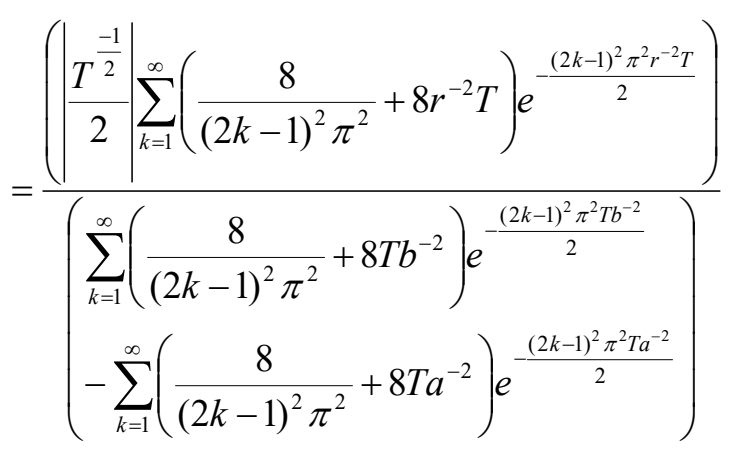

where $a<r<$ band $T>0$ (see Appendix A).

Figure 4 represents the TDWR density function for different values of $a$ and $b$ with increasing the value of $T$.

Remark 1. Using the ratio test, we can prove that $\sum_{k=1}^{\infty} \frac{8 e^{-\frac{(2 k-1)^{2} \pi^{2} r^{-2} T}{2}}}{(2 k-1)^{2} \pi^{2}}$ is convergent where $\lim _{k \rightarrow \infty}\left[\sum_{k=1}^{\infty} \frac{8 e^{-\frac{(2(k+1)-1)^{2} \pi^{2} r^{-2} T}{2}}}{(2(k+1)-1)^{2} \pi^{2}} / \sum_{k=1}^{\infty} \frac{8 e^{-\frac{(2 k-1)^{2} \pi^{2} r^{-2} T}{2}}}{(2 k-1)^{2} \pi^{2}}\right]=0$. Thus, by the Weierstrass M-Test we see that, $\sum_{k=1}^{\infty} \frac{8 e^{-\frac{(2 k-1)^{2} \pi^{2} r^{-2} T}{2}}}{(2 k-1)^{2} \pi^{2}}$ is uniformly convergent to 0 . Consequently, we can get (1) from (3) when $a \rightarrow 0$ and $b \rightarrow \infty$. 


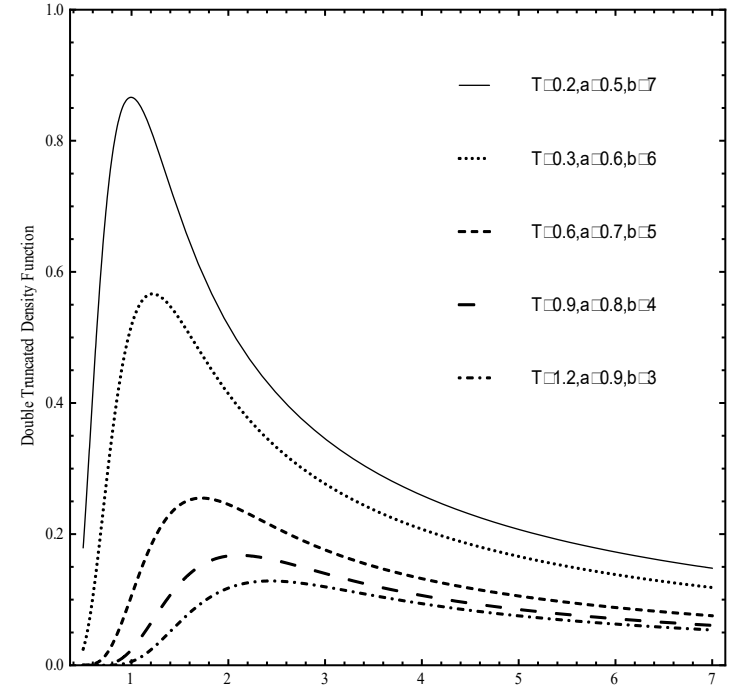

Figure 4: Double truncated probability density

The cumulative distribution function of TDWR is given by:

$$
G_{R(T)}(r)=\int_{a}^{r} \frac{f_{\bar{R}(T)}(r)}{F_{\bar{R}(T)}(b)-F_{\bar{R}(T)}(a)} d r,
$$

using integration by parts as in the appendix A, we get:

$$
G_{R(T)}(r)=\frac{\left(\left|\frac{T^{\frac{-1}{2}}}{2}\right| \sum_{k=1}^{\infty} \frac{8\left(-a e^{\frac{-(1-2 k)^{2} \pi^{2} T}{2 a^{2}}}+r e^{\frac{-(1-2 k)^{2} \pi^{2} T}{2 r^{2}}}\right)}{(\pi-2 k \pi)^{2}}\right)}{\left(\begin{array}{l}
\sum_{k=1}^{\infty}\left(\frac{8}{(2 k-1)^{2} \pi^{2}}+8 T b^{-2}\right) e^{-\frac{(2 k-1)^{2} \pi^{2} b^{-2}}{2}} \\
-\sum_{k=1}^{\infty}\left(\frac{8}{(2 k-1)^{2} \pi^{2}}+8 T a^{-2}\right) e^{-\frac{(2 k-1)^{2} \pi^{2} T a^{-2}}{2}}
\end{array}\right),}
$$

and it is represented in figure 5.

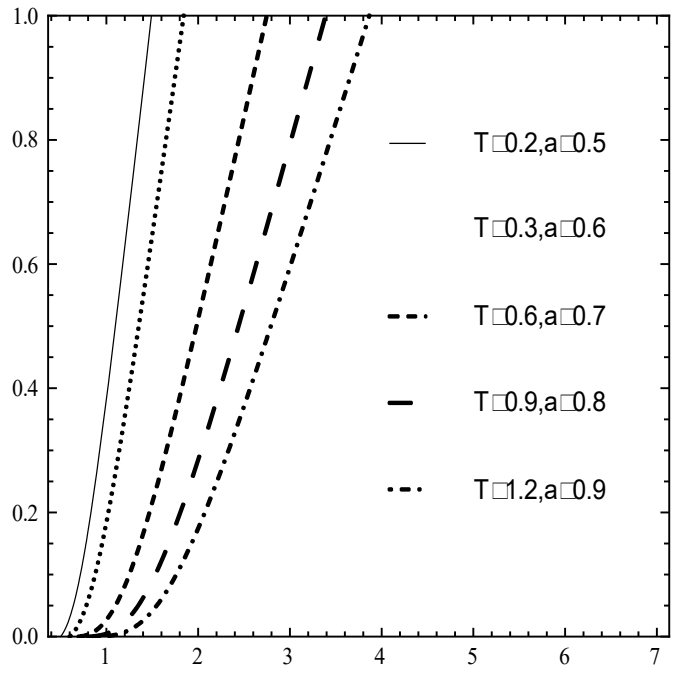

Figure 5: Cumulative distribution function of TDWR.

Consequently, the survival function of TDWR is $\bar{G}_{R(T)}()=.1-G_{R(T)}(r)$ and it is decreasing by increasing the value of $T$, see figure 6 .

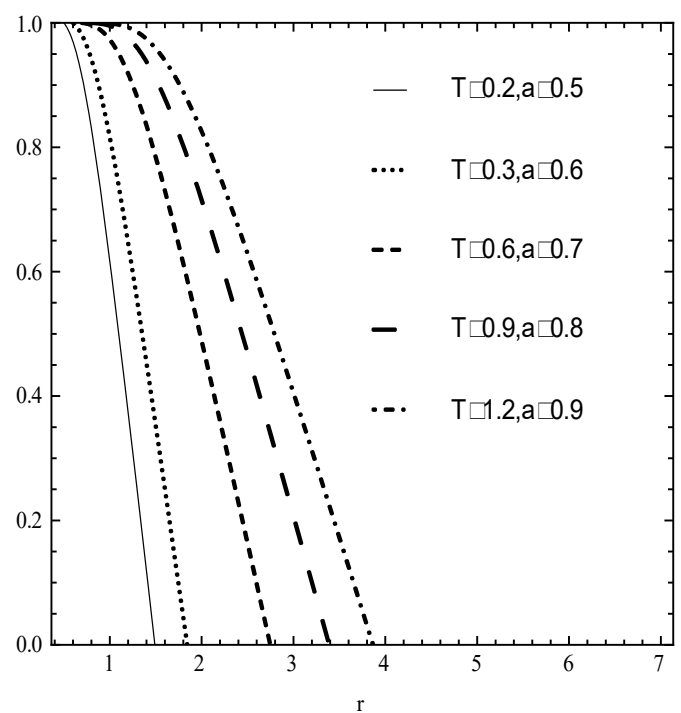

Figure 6:Survival function of the TDWR.

\section{Some statistical properties}

In this section, we study various statistical properties of the range distribution (truncated and non truncated) including shapes of the probability distribution function and the hazard rate function, reliability properties, 
raw moments, moments of (reversed) residual life, stress-strength parameter, Bonferroni curve, Lorenz curve and Gini's index.

\subsection{Reliability properties}

A key concept of "Whenever you want to check more than one investment profits in the stock market, investment whenever exposed to greater risk." You are when you buy or sell shares or bonds or any other financial instruments, you are fair investment risk and the degree of risk this differ from other financial instrument. For example, the financial instruments that you expect them highly profitable (such as active stock) contain a large degree of risk. This means that the share price could rise so much (that is to make a profit for you), but it may happen that the price drops much (and these are the risks that may cause the low volume of your money and your investments). Therefore, the risk rate (hazard rate) is influenced by the swings between fall and rise much of the stock price during the time period $(0, T)$. We get the hazard rate function of the range distribution for Feller [1] and Withers and Nadarajah [2] as follows:

$$
\begin{aligned}
& z_{\bar{R}(T)}(\bar{r})=f_{\bar{R}(T)}\left\{\bar{F}_{\bar{R}(t)}(\bar{r})\right\}^{-1} \\
& =\frac{\left(\frac{2}{\pi}\right)^{\frac{1}{2}} \bar{r}^{-1}(2 \pi)^{\frac{1}{2}}\left(\frac{\bar{r} T^{-\frac{1}{2}}}{2}\right)^{-1} \sum_{k=1}^{\infty} \exp \left[-\frac{(2 k-1)^{2} \pi^{2}}{8} \cdot\left(\frac{\bar{r} T^{-\frac{1}{2}}}{2}\right)^{-2}\right]}{1-\sum_{k=1}^{\infty}\left(\frac{8}{(2 k-1)^{2} \pi^{2}}+\frac{8 T}{\bar{r}^{2}}\right) \exp \left[-\frac{(2 k-1)^{2} \pi^{2} T}{2 \bar{r}^{2}}\right]},
\end{aligned}
$$

and it is represented as in figure 7. Also, the reversed hazard rate function is:

$$
\begin{aligned}
& \widetilde{z}_{\bar{R}(T)}(\bar{r})=f_{\bar{R}(T)}\left\{F_{\bar{R}(t)}(\bar{r})\right\}^{-1} \\
& =\frac{\left(\frac{2}{\pi}\right)^{\frac{1}{2}} \bar{r}^{-1}(2 \pi)^{\frac{1}{2}}\left(\frac{\bar{r} T^{-\frac{1}{2}}}{2}\right)^{-1} \sum_{k=1}^{\infty} \exp \left[-\frac{(2 k-1)^{2} \pi^{2}}{8} \cdot\left(\frac{\bar{r} T^{-\frac{1}{2}}}{2}\right)^{-2}\right]}{1-\sum_{k=1}^{\infty}\left(\frac{8}{(2 k-1)^{2} \pi^{2}}+\frac{8 T}{\bar{r}^{2}}\right) \exp \left[-\frac{(2 k-1)^{2} \pi^{2} T}{2 \bar{r}^{2}}\right]},
\end{aligned}
$$

See figure 8.

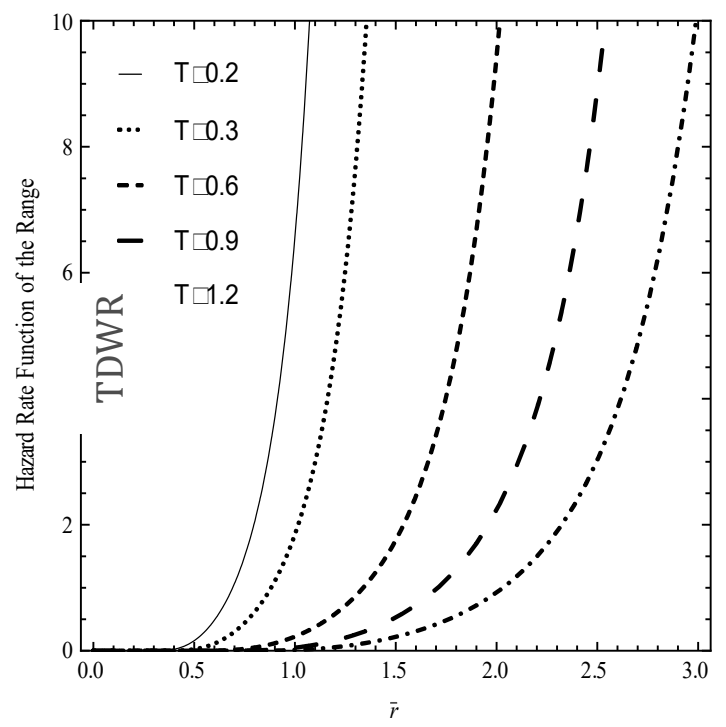

Figure 7: Hazard rate function of the range.

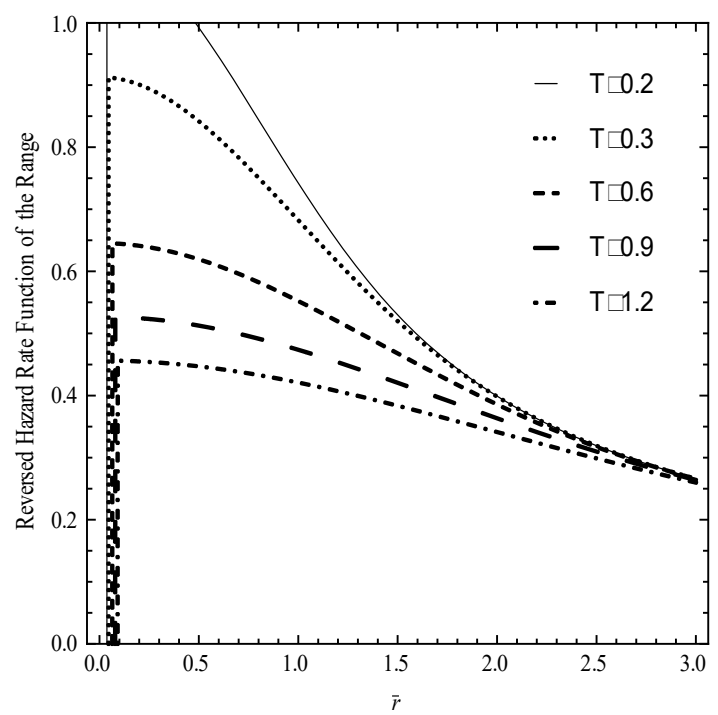

Figure 8: Reversed hazard rate function of the range.

It is clear that, the hazard rate approaches zero as the range $\bar{r}$ increases, and increases rapidly as $\bar{r}$ falls to zero. For the new distribution of TDWR, the hazard rate function is:

$$
\Theta_{R(T)}(r)=g_{R(T)}\left\{\bar{G}_{R(t)}(r)\right\}^{-1},
$$

and it is represented in figure 9. 


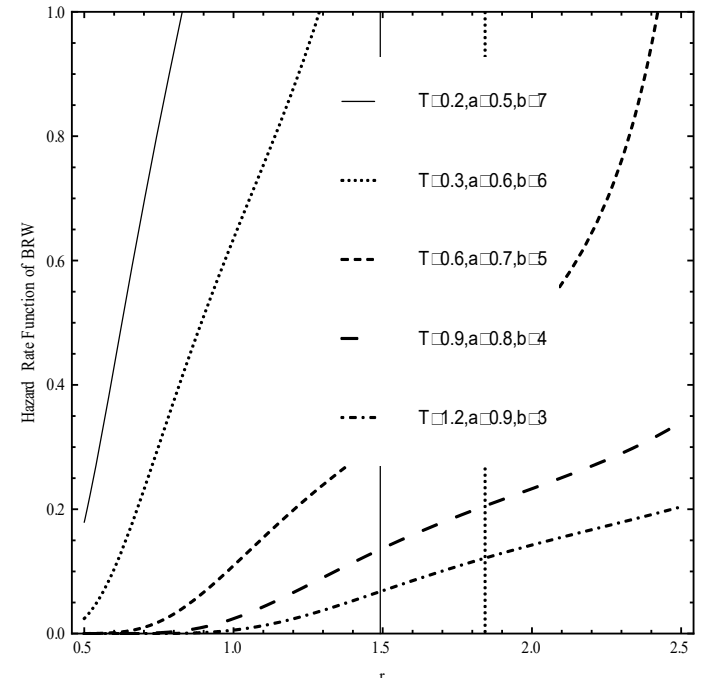

Figure 9: Hazard rate function of TDWR.

In addition, the reversed hazard rate function of TDWR is:

$$
\widetilde{\Theta}_{R(T)}(r)=g_{R(T)}\left\{G_{R(t)}(r)\right\}^{-1},
$$

see figure 10. Also, in this case the hazard rate increases rapidly as $r$ falls to lower bound $a$.

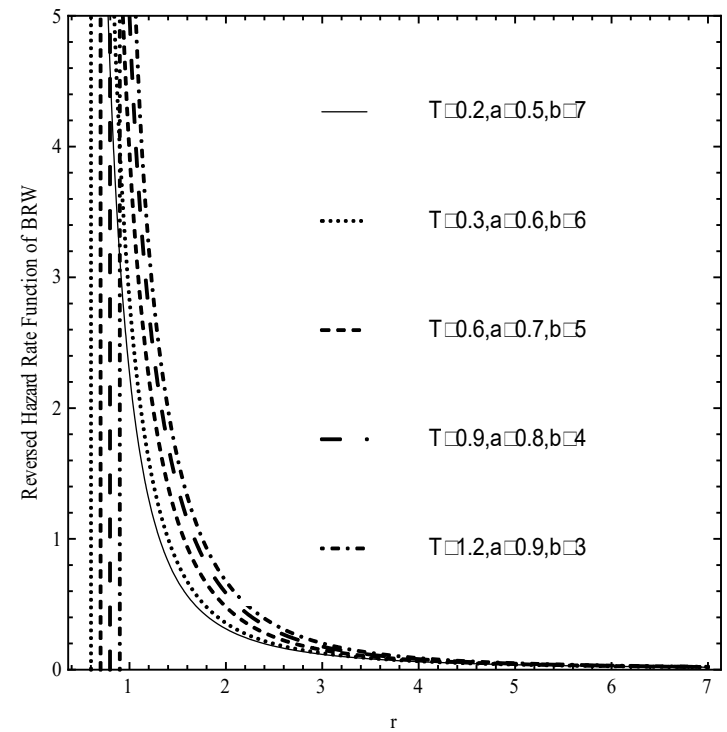

Figure 10: Reversed hazard rate function

\subsection{Moments}

Many interesting characteristics and features of the range distribution and TDWR can be studied through its generating function and moments. For the range distribution (1) Withers and Nadarajah [2] found its generating, characteristic functions and moments. Here, if $R$ has TDWR distribution and $a<r<b$ and $T>0$ then the moment generating function (m.g.f.) of $R$ defined by:

$$
\begin{aligned}
& M(t)=E\left(e^{t r}\right) \\
& =\int_{a}^{b} \frac{\left(\frac{T^{\frac{-1}{2}}}{2} \mid \sum_{k=1}^{\infty}\left(\frac{8}{(2 k-1)^{2} \pi^{2}}+8 r^{-2} T\right) e^{-\frac{(2 k-1)^{2} \pi^{2} r^{-2} T}{2}}\right) e^{t r}}{\left(\sum_{k=1}^{\infty}\left(\frac{8}{(2 k-1)^{2} \pi^{2}}+8 T b^{-2}\right) e^{-\frac{(2 k-1)^{2} \pi^{2} T b^{-2}}{2}}\right)} d r \\
& \left.-\sum_{k=1}^{\infty}\left(\frac{8}{(2 k-1)^{2} \pi^{2}}+8 T a^{-2}\right) e^{-\frac{(2 k-1)^{2} \pi^{2} T a^{-2}}{2}}\right) \\
& =\frac{\left(\mid \frac{T^{\frac{-1}{2}}}{2} I(a, b, t, T)\right)}{\left(\sum_{k=1}^{\infty}\left(\frac{8}{(2 k-1)^{2} \pi^{2}}+8 T b^{-2}\right) e^{-\frac{(2 k-1)^{2} \pi^{2} T b^{-2}}{2}}\right)} \\
& \left.-\sum_{k=1}^{\infty}\left(\frac{8}{(2 k-1)^{2} \pi^{2}}+8 T a^{-2}\right) e^{-\frac{(2 k-1)^{2} \pi^{2} T a^{-2}}{2}}\right)
\end{aligned}
$$

where,

$$
I(a, b, t, T)=\int_{a}^{b} \sum_{k=1}^{\infty}\left(\alpha_{k}+\frac{\hat{a}}{r^{2}}\right) e^{\left(t r-\frac{\beta_{k}}{r^{2}}\right)} d r,
$$

and

$\alpha_{k}=\frac{8}{(2 k-1)^{2} \pi^{2}}, \hat{a}=8 T, \quad \beta_{k}=\frac{(2 k-1)^{2} \pi^{2} T}{2}$. Since the expansion of the exponential function is valid for $r \in(-\infty, \infty)$ and the series is uniformly convergent, then we have

$$
\begin{aligned}
e^{\left(t r-\frac{\beta_{k}}{r^{2}}\right)} & =\sum_{m=0}^{\infty} \frac{e^{\left(t r-\frac{\beta_{k}}{r^{2}}\right)^{m}}}{m !}=1+t r-\frac{\beta_{k}}{r^{2}}+\sum_{m=2}^{\infty} \frac{e^{\left(t r-\frac{\beta_{k}}{r^{2}}\right)^{m}}}{m !} \\
= & 1+t r-\frac{\beta_{k}}{r^{2}}+\sum_{m=2}^{\infty} \sum_{\mu=0}^{m} \frac{t^{m-\mu}}{m !}\left(\begin{array}{l}
m \\
\mu
\end{array}\right)\left(-\beta_{k}\right)^{\mu} r^{m-3 \mu} .
\end{aligned}
$$

Assume that,

$I(a, b, t, T)=I_{1}(a, b, t, T)+I_{2}(a, b, t, T)$

where, 
$I_{1}(a, b, t, T)=\sum_{k=1}^{\infty} \int_{a}^{b} \alpha_{k} e^{\left(t r-\frac{\beta_{k}}{r^{2}}\right)} d r$

$=\sum_{k=1}^{\infty} \int_{a}^{b} \alpha_{k}\left(1+t r-\frac{\beta_{k}}{r^{2}}+\sum_{m=2}^{\infty} \sum_{\mu=0}^{m} \frac{t^{m-\mu}}{m !}\left(\begin{array}{l}m \\ \mu\end{array}\right)\left(-\beta_{k}\right)^{\mu} r^{m-3 \mu}\right) d r$

and

$$
\begin{aligned}
& I_{2}(a, b, t, T)=\sum_{k=1}^{\infty} \int_{a}^{b} \frac{\hat{a}}{r^{2}} e^{\left(t r-\frac{\beta_{k}}{r^{2}}\right)} d r \\
& =\hat{a} \sum_{k=1}^{\infty} \int_{a}^{b}\left(1+t r-\frac{\beta_{k}}{r^{2}}+\sum_{m=2}^{\infty} \sum_{\mu=0}^{m} \frac{t^{m-\mu}}{m !}\left(\begin{array}{c}
m \\
\mu
\end{array}\right)\left(-\beta_{k}\right)^{\mu} r^{m-2-3 \mu}\right) d r .
\end{aligned}
$$

By solving the following equations:

$$
\begin{aligned}
& m-3 \mu=-1, \\
& m-2-3 \mu=-1,
\end{aligned}
$$

as Diophantine equations, we have the set solution for (11) is given by:

$$
S_{1}=\left(m_{S_{1}}, \mu_{S_{1}}\right)=\{(2,1),(5,2),(8,3),(11,4), \ldots\} \text {, }
$$

and for (12)

$$
S_{2}=\left(m_{S_{2}}, \mu_{S_{2}}\right)=\{(4,1),(7,2),(10,3),(13,4), \ldots\} \text {. }
$$

Hence, $I_{1}(a, b, t, T)$ can be written as follows:

$$
\begin{aligned}
& I_{1}(a, b, t, T)=\sum_{k=1}^{\infty} \alpha_{k}\left[(b-a)+\frac{t}{2}\left(b^{2}-a^{2}\right)-\beta_{k}\left(\frac{1}{a}-\frac{1}{b}\right)\right] \\
&+ \sum_{k=1}^{\infty} \alpha_{k}\left[\begin{array}{l}
\forall m,\left(\mu \neq \mu_{S_{1}} \wedge m=m_{S_{1}}\right) \\
{\left[\sum_{m=2}^{\infty}\left(\begin{array}{l}
m \\
\mu=0
\end{array}\right) \frac{\left(-\beta_{k}\right)^{\mu} t^{m-\mu}}{m !(m+1-3 \mu)}\left(b^{m+1-3 \mu}-a^{m+1-3 \mu}\right)\right]}
\end{array}\right. \\
&\left.+\sum_{k=1}^{\infty} \alpha_{k} \sum_{(m, \mu) \in S_{1}}\left(\begin{array}{l}
m \\
\mu
\end{array}\right) \frac{\left(-\beta_{k}\right)^{\mu}}{m !} t^{m-\mu} \ln \frac{b}{a}\right),
\end{aligned}
$$

Also, $I_{2}(a, b, t, T)$ can be written as follows:

$$
\begin{aligned}
& I_{2}(a, b, t, T)=\hat{a} \sum_{k=1}^{\infty}\left[\left(a^{-1}-b^{-1}\right)+t \ln \frac{b}{a}-\frac{\beta_{k}}{3}\left(\frac{1}{a^{3}}-\frac{1}{b^{3}}\right)\right] \\
& +\hat{a} \sum_{k=1}^{\infty} \overbrace{\left[\sum_{m=2}^{\infty} \sum_{\mu=0}^{m}\left(\begin{array}{l}
m \\
\mu
\end{array}\right) \frac{\left(-\beta_{k}\right)^{\mu} t^{m-\mu}}{m !(m-1-3 \mu)}\left(b^{m-1-3 \mu}-a^{m-1-3 \mu}\right)\right]}+\hat{a} \sum_{k=1}^{\infty}\left(\sum_{(m, \mu) \in S_{2}}\left(\begin{array}{l}
m \\
\mu
\end{array}\right) \frac{\left(-\beta_{k}\right)^{\mu}}{m !} t^{m-\mu} \ln \frac{b}{a}\right) .
\end{aligned}
$$

Consequently, from (15) and (16) we obtain the value of (8). Hence,

$$
\begin{aligned}
& M(t)=E\left(e^{t r}\right) \\
& =\int_{a}^{b} \frac{\left(\mid \frac{T^{\frac{-1}{2}}}{2} \sum_{k=1}^{\infty}\left(\frac{8}{(2 k-1)^{2} \pi^{2}}+8 r^{-2} T\right) e^{-\frac{(2 k-1)^{2} \pi^{2} r^{-2} T}{2}}\right) e^{t r}}{\left(\sum_{k=1}^{\infty}\left(\frac{8}{(2 k-1)^{2} \pi^{2}}+8 T b^{-2}\right) e^{-\frac{(2 k-1)^{2} \pi^{2} T b^{-2}}{2}}\right)} d r \\
& =\frac{\left.-\sum_{k=1}^{\infty}\left(\frac{8}{(2 k-1)^{2} \pi^{2}}+8 T a^{-2}\right) e^{-\frac{(2 k-1)^{2} \pi^{2} T a^{-2}}{2}}\right)}{\left(\mid \frac{T^{\frac{-1}{2}}}{2}\left(I_{1}(a, b, t, T)+I_{2}(a, b, t, T)\right)\right)} . \\
& \left(\begin{array}{l}
\sum_{k=1}^{\infty}\left(\frac{8}{(2 k-1)^{2} \pi^{2}}+8 T b^{-2}\right) e^{-\frac{(2 k-1)^{2} \pi^{2} T b^{-2}}{2}} \\
\left.-\sum_{k=1}^{\infty}\left(\frac{8}{(2 k-1)^{2} \pi^{2}}+8 T a^{-2}\right) e^{-\frac{(2 k-1)^{2} \pi^{2} T a^{-2}}{2}}\right)
\end{array}\right.
\end{aligned}
$$

Also, the moments of $R$ about the origin can be obtained as:

$$
E\left(r^{q}\right)=\int_{a}^{b} r^{q} g_{R(T)}(r) d r .
$$

To solve (18), we let, $x=r T^{-\frac{1}{2}} / 2$ and $\omega_{k}=(2 k-1)^{2} \pi^{2} / 8$ then (3) become,

$$
\Psi_{T}(x)=\frac{1}{\wp}\left|\frac{T^{\frac{-1}{2}}}{2}\right| \sum_{k=1}^{\infty}\left[\begin{array}{c}
\left(-4 x^{-3} \exp \left(-\omega_{k} x^{-2}\right)\right) \\
+\left(2 \omega_{k} x^{-3} \exp \left(-\omega_{k} x^{-2}\right)\right) \\
\times\left(\omega_{k}^{-1}+2 x^{-2}\right)
\end{array}\right] \text {, }
$$

where,

$$
\begin{aligned}
\wp & =\sum_{k=1}^{\infty}\left(\frac{8}{(2 k-1)^{2} \pi^{2}}+8 T b^{-2}\right) e^{-\frac{(2 k-1)^{2} \pi^{2} T b^{-2}}{2}} \\
& -\sum_{k=1}^{\infty}\left(\frac{8}{(2 k-1)^{2} \pi^{2}}+8 T a^{-2}\right) e^{-\frac{(2 k-1)^{2} \pi^{2} T a^{-2}}{2}} .
\end{aligned}
$$

Since the series, 


$$
\sum_{k=1}^{\infty}\left[\begin{array}{l}
\left(-4 x^{-3} \exp \left(-\omega_{k} x^{-2}\right)\right) \\
+\left(2 \omega_{k} x^{-3} \exp \left(-\omega_{k} x^{-2}\right)\right)\left(\omega_{k}^{-1}+2 x^{-2}\right)
\end{array}\right]
$$

is uniformly convergence series, then we get,

$$
E\left(x^{q}\right)=\frac{1}{\wp} \sum_{k=1}^{\infty} \Delta_{k},
$$

where,

$$
\Delta_{k}=\int_{a}^{b} x^{q}\left[\begin{array}{c}
\left(-4 x^{-3} \exp \left(-\omega_{k} x^{-2}\right)\right) \\
+\left(2 \omega_{k} x^{-3} \exp \left(-\omega_{k} x^{-2}\right)\right) \\
\times\left(\omega_{k}^{-1}+2 x^{-2}\right)
\end{array}\right] d x .
$$

Thus,

$$
\begin{aligned}
\Delta_{k}= & a^{-2+q} \xi\left[\frac{q}{2}, \frac{\omega_{k}}{a^{2}}\right]-b^{-2+q} \xi\left[\frac{q}{2}, \frac{\omega_{k}}{b^{2}}\right] \\
& +2 \omega_{k}\left[\begin{array}{l}
a^{-4+q} \xi\left[-1+\frac{q}{2}, \frac{\omega_{k}}{a^{2}}\right] \\
\left.+b^{-4+q} \xi\left[-1+\frac{q}{2}, \frac{\omega_{k}}{b^{2}}\right]\right]
\end{array}\right.
\end{aligned}
$$

where $\xi$ is the exponential integral function.

Consequently,

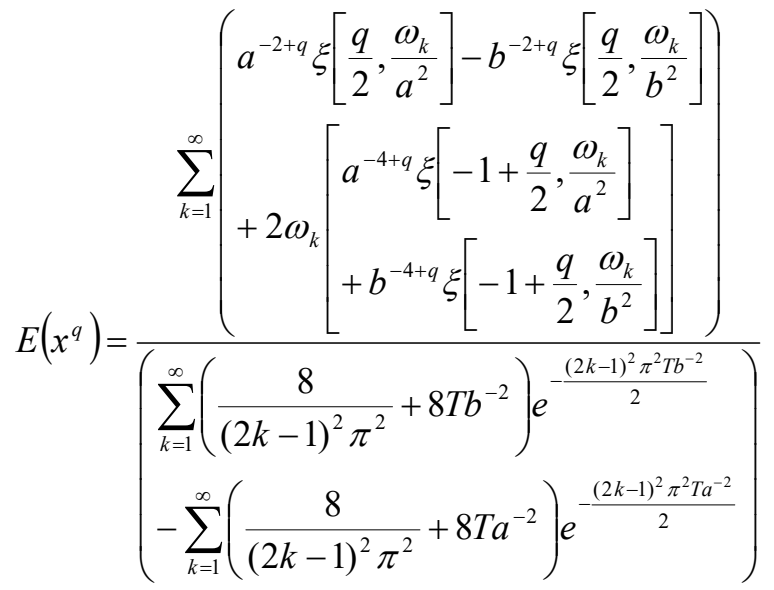

By substation again with $x=r T^{-\frac{1}{2}} / 2$ and $\omega_{k}=(2 k-1)^{2} \pi^{2} / 8$ then we get:

$$
E\left(r^{q}\right)=\frac{2^{q} T^{\frac{q}{2}} \sum_{k=1}^{\infty}\left(\begin{array}{l}
a^{-2+q} \xi\left[\frac{q}{2}, \frac{(2 k-1)^{2} \pi^{2}}{8 a^{2}}\right] \\
\left.-b^{-2+q} \xi\left[\frac{q}{2}, \frac{(2 k-1)^{2} \pi^{2}}{8 b^{2}}\right]+\lambda_{k}\right)
\end{array}\right.}{\left(\begin{array}{l}
\sum_{k=1}^{\infty}\left(\frac{8}{(2 k-1)^{2} \pi^{2}}+8 T b^{-2}\right) e^{-\frac{(2 k-1)^{2} \pi^{2} T b^{-2}}{2}} \\
-\sum_{k=1}^{\infty}\left(\frac{8}{(2 k-1)^{2} \pi^{2}}+8 T a^{-2}\right) e^{-\frac{(2 k-1)^{2} \pi^{2} T a^{-2}}{2}}
\end{array}\right)}
$$

where,

$\lambda_{k}=\frac{(2 k-1)^{2} \pi^{2}}{4}\left[\begin{array}{l}a^{-4+q} \xi\left[-1+\frac{q}{2}, \frac{(2 k-1)^{2} \pi^{2}}{8 a^{2}}\right] \\ +b^{-4+q} \xi\left[-1+\frac{q}{2}, \frac{(2 k-1)^{2} \pi^{2}}{8 b^{2}}\right]\end{array}\right]$.

In addition, the characteristic function can get from the equation:

$$
\begin{aligned}
& \hat{M}(t)=E\left(e^{i t r}\right) \\
& =\int_{a}^{b} \frac{\left(\left|\frac{T^{\frac{-1}{2}}}{2}\right| \sum_{k=1}^{\infty}\left(\frac{8}{(2 k-1)^{2} \pi^{2}}+8 r^{-2} T\right) e^{-\frac{(2 k-1)^{2} \pi^{2} r^{-2} T}{2}}\right) e^{i t r}}{\left(\sum_{k=1}^{\infty}\left(\frac{8}{(2 k-1)^{2} \pi^{2}}+8 T b^{-2}\right) e^{-\frac{(2 k-1)^{2} \pi^{2} T b^{-2}}{2}}\right)} d r \\
& =\frac{\left.-\sum_{k=1}^{\infty}\left(\frac{8}{(2 k-1)^{2} \pi^{2}}+8 T a^{-2}\right) e^{-\frac{(2 k-1)^{2} \pi^{2} T a^{-2}}{2}}\right)}{\left(\mid \frac{T^{\frac{-1}{2}}}{2}\left(\left(\hat{I}_{1}(a, b, t, T)+\hat{I}_{2}(a, b, t, T)\right)\right.\right.} \\
& \left(\begin{array}{l}
\left.\sum_{k=1}^{\infty}\left(\frac{8}{(2 k-1)^{2} \pi^{2}}+8 T b^{-2}\right) e^{-\frac{(2 k-1)^{2} \pi^{2} T b^{-2}}{2}}\right) \\
\left.-\sum_{k=1}^{\infty}\left(\frac{8}{(2 k-1)^{2} \pi^{2}}+8 T a^{-2}\right) e^{-\frac{(2 k-1)^{2} \pi^{2} T a^{-2}}{2}}\right)
\end{array}\right.
\end{aligned}
$$

where, $i=\sqrt{-1}$, 
$\hat{I}_{1}(a, b, t, T)=\sum_{k=1}^{\infty} \alpha_{k}\left[\begin{array}{l}(b-a)+\frac{i t}{2}\left(b^{2}-a^{2}\right) \\ -\beta_{k}\left(\frac{1}{a}-\frac{1}{b}\right)\end{array}\right] \quad \begin{array}{lrrr}\text { And, for } & \operatorname{TDWR}\left(T_{1}\right) & \text { and } & \operatorname{TDWR}\left(T_{2}\right) \\ \text { distribution, } & \text { we } & \text { find } & \text { that } \\ \tilde{Y}=P\left(R\left(T_{2}\right)<R\left(T_{1}\right)\right) & \text { where } & \widetilde{Y} \text { has }\end{array}$ $\forall m,\left(\mu \neq \mu_{S_{1}} \wedge m=m_{S_{1}}\right)$

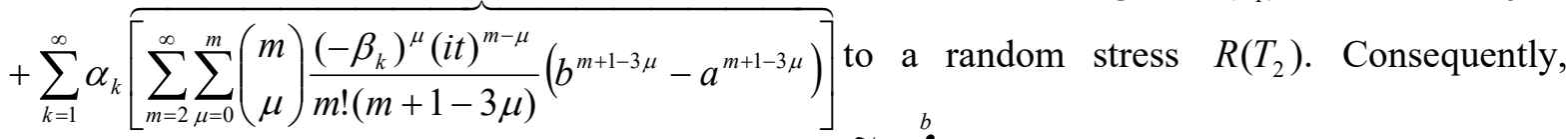
$+\sum_{k=1}^{\infty} \alpha_{k}\left(\sum_{(m, \mu) \in S_{1}}\left(\begin{array}{l}m \\ \mu\end{array}\right) \frac{\left(-\beta_{k}\right)^{\mu}}{m !}(i t)^{m-\mu} \ln \frac{b}{a}\right)$,

$\widetilde{Y}=\int_{a}^{b} G_{R(T)}\left(r ; T_{2}\right) \cdot g_{R(T)}\left(r ; T_{1}\right) d r$. Now we find and

$$
\begin{aligned}
& \hat{I}_{2}(a, b, t, T)=\hat{a} \sum_{k=1}^{\infty}\left[\begin{array}{l}
\left(a^{-1}-b^{-1}\right) \\
+i t \ln \frac{b}{a}-\frac{\beta_{k}}{3}\left(\frac{1}{a^{2}}-\frac{1}{b^{2}}\right)
\end{array}\right] \\
& +\hat{a} \sum_{k=1}^{\infty} \overbrace{\left[\sum_{m=2}^{\infty} \sum_{\mu=0}^{m}\left(\begin{array}{c}
m \\
\mu
\end{array}\right) \frac{\left(-\beta_{k}\right)^{\mu}(i t)^{m-\mu}}{m !(m-1-3 \mu)}\left(b^{m-1-3 \mu}-a^{m-1-3 \mu}\right)\right]}^{\forall m,\left(\mu \neq \mu_{S_{2}} \wedge m=m_{S_{2}}\right)} \\
& +\hat{a} \sum_{k=1}^{\infty}\left(\sum_{(m, \mu) \in S_{2}}\left(\begin{array}{c}
m \\
\mu
\end{array}\right) \frac{\left(-\beta_{k}\right)^{\mu}}{m !}(i t)^{m-\mu} \ln \frac{b}{a}\right) \text {. }
\end{aligned}
$$

$\widetilde{Y}$ by assuming that,

\subsection{Stress-strength parameter}

In this section, we find $Y=P\left(\bar{R}\left(T_{2}\right)<\bar{R}\left(T_{1}\right)\right)$, when $\quad \bar{R}\left(T_{1}\right) \quad$ and $\bar{R}\left(T_{2}\right)$ are two independent random variables distributed as in (1) with $T_{1}, T_{2}$, respectively. In the statistical literature $Y$ is known as the stress-strength parameter which describes the changing of stock price. In addition, $Y$ has a random strength $\bar{R}\left(T_{1}\right)$ that is subject to a random stress $\bar{R}\left(T_{2}\right)$. The changing in stock price at the instant that the stress applied to it exceeds the strength, and the changing will function satisfactorily whenever $\bar{R}\left(T_{2}\right)>\bar{R}\left(T_{1}\right)$; see, for example, Church and

$$
\begin{aligned}
Q_{1}= & \sum_{k=1}^{\infty}\left(\frac{8}{(2 k-1)^{2} \pi^{2}}+8 T_{1} b^{-2}\right) e^{-\frac{(2 k-1)^{2} \pi^{2} T b^{-2}}{2}} \\
& -\sum_{k=1}^{\infty}\left(\frac{8}{(2 k-1)^{2} \pi^{2}}+8 T_{1} a^{-2}\right) e^{-\frac{(2 k-1)^{2} \pi^{2} T_{1} a^{-2}}{2}} \\
Q_{2}= & \sum_{k=1}^{\infty}\left(\frac{8}{(2 k-1)^{2} \pi^{2}}+8 T_{2} b^{-2}\right) e^{-\frac{(2 k-1)^{2} \pi^{2} T_{2} b^{-2}}{2}} \\
& -\sum_{k=1}^{\infty}\left(\frac{8}{(2 k-1)^{2} \pi^{2}}+8 T_{2} a^{-2}\right) e^{-\frac{(2 k-1)^{2} \pi^{2} T_{2} a^{-2}}{2}}, \\
C_{k}= & \frac{-8 a}{(\pi-2 k \pi)^{2}}, \quad B_{k}=\frac{8}{(\pi-2 k \pi)^{2}}
\end{aligned}
$$

$N_{k}=\frac{(1-2 k)^{2} \pi^{2}}{2}, D_{L}=\frac{8}{(2 L-1)^{2} \pi^{2}}$ and

$F_{L}=\frac{(2 L-1)^{2} \pi^{2}}{2}$. Then,

$$
\begin{aligned}
& \tilde{Y}=\frac{\left|\frac{T_{1}^{-\frac{1}{2}}}{2}\right|\left|\frac{T_{2}^{-\frac{1}{2}}}{2}\right|}{Q_{1} Q_{2}} \\
& \times \sum_{k=1}^{\infty} \sum_{L=1}^{\infty} \int_{a}^{b}\left[\left(\begin{array}{l}
\left(C_{k} e^{-N_{k} a^{-2} T_{2}}+B_{k} r e^{-N_{k} r^{-2} T_{2}}\right) \\
\left.\times\left(D_{L} e^{-F_{L} r^{-2} T_{1}}+8 r^{-2} T_{1} e^{-F_{l} r^{-2} T_{1}}\right)\right] d r
\end{array} .\right.\right.
\end{aligned}
$$

Let, Harris [6]. Thus, for the range distribution, $Y$ can be expressed as:

$$
Y=\int_{0}^{\infty} F_{\bar{R}(T)}\left(\bar{r} ; T_{2}\right) \cdot f_{\bar{R}(T)}\left(\bar{r} ; T_{1}\right) d \bar{r}
$$




$$
\begin{aligned}
& J\left(a, b, T_{1}, T_{2}\right)=\int_{a}^{b}\left[\begin{array}{l}
\left(C_{k} e^{-N_{k} a^{-2} T_{2}}+B_{k} r e^{-N_{k} r^{-2} T_{2}}\right) \\
\times\left(D_{L} e^{-F_{L} r^{-2} T_{1}}+8 r^{-2} T_{1} e^{-F_{l} r^{-2} T_{1}}\right)
\end{array}\right] d r \\
& =\int_{a}^{b}\left(\begin{array}{l}
C_{k} e^{-N_{k} a^{-2} T_{2}} D_{L} e^{-F_{L} r^{-2} T_{1}} \\
+C_{k} e^{-N_{k} a^{-2} T_{2}} 8 r^{-2} T_{1} e^{-F_{l} r^{-2} T_{1}} \\
+B_{k} r e^{-N_{k} r^{-2} T_{2}} D_{L} e^{-F_{L} r^{-2} T_{1}} \\
+B_{k} r e^{-N_{k} r^{-2} T_{2}} 8 r^{-2} T_{1} e^{-F_{l} r^{-2} T_{1}}
\end{array}\right) d r \\
& =C_{k} e^{-N_{k} a^{-2} T_{2}} D_{L} \int_{a}^{b} e^{-F_{L} r^{-2} T_{1}} d r \\
& +C_{k} e^{-N_{k} a^{-2} T_{2}} 8 T_{1} \int_{a}^{b} r^{-2} e^{-F_{l} r^{-2} T_{1}} d r \\
& +B_{k} D_{L} \int_{a}^{b} r e^{-N_{k} r^{-2} T_{2}} e^{-F_{L} r^{-2} T_{1}} d r \\
& +B_{k} 8 T_{1} \int_{a}^{b} r^{-1} e^{-N_{k} r^{-2} T_{2}} e^{-F_{l} r^{-2} T_{1}} d r . \\
& =C_{k} e^{-N_{k} a^{-2} T_{2}} D_{L} J_{1}\left(a, b, T_{1}, T_{2}\right) \\
& +C_{k} e^{-N_{k} a^{-2} T_{2}} 8 T_{1} J_{2}\left(a, b, T_{1}, T_{2}\right) \\
& +B_{k} D_{L} J_{3}\left(a, b, T_{1}, T_{2}\right) \\
& +B_{k} 8 T_{1} J_{4}\left(a, b, T_{1}, T_{2}\right) \text {, }
\end{aligned}
$$$$
J_{3}\left(a, b, T_{1}, T_{2}\right)=\int_{a}^{b} r e^{-N_{k} r^{-2} T_{2}} e^{-F_{L} r^{-2} T_{1}} d r
$$$$
=\frac{1}{2} F_{L} T_{1}\left(\begin{array}{l}
\Gamma\left(0, \frac{F_{L} T_{1}+N_{K} T_{2}}{a^{2}}\right) \\
-\Gamma\left(0, \frac{F_{L} T_{1}+N_{K} T_{2}}{b^{2}}\right)
\end{array}\right)
$$$$
+\frac{1}{2}\left(\begin{array}{c}
-a^{2} e^{-\frac{F_{L} T_{1}+N_{K} T_{2}}{a^{2}}}+b^{2} e^{-\frac{F_{L} T_{1}+N_{K} T_{2}}{b^{2}}} \\
+N_{K} T_{2}\left(\begin{array}{c}
\Gamma\left(0, \frac{F_{L} T_{1}+N_{K} T_{2}}{a^{2}}\right) \\
-\Gamma\left(0, \frac{F_{L} T_{1}+N_{K} T_{2}}{b^{2}}\right)
\end{array}\right)
\end{array}\right) ;
$$

and

$$
\begin{aligned}
J_{4}\left(a, b, T_{1}, T_{2}\right)= & \int_{a}^{b} r^{-1} e^{-N_{k} r^{-2} T_{2}} e^{-F_{l} r^{-2} T_{1}} d r \\
& \frac{1}{2}\left(\begin{array}{l}
\left.-\Gamma\left(0, \frac{F_{L} T_{1}+N_{K} T_{2}}{a^{2}}\right)\right) \\
+\Gamma\left(0, \frac{F_{L} T_{1}+N_{K} T_{2}}{b^{2}}\right)
\end{array}\right)
\end{aligned}
$$

Consequently,

where,

$$
\begin{array}{r}
J_{1}\left(a, b, T_{1}, T_{2}\right)=\int_{a}^{b} e^{-F_{L} r^{-2} T_{1}} d r=-a e^{-\frac{F_{l} T_{1}}{a^{2}}}+b e^{-\frac{F_{l} T_{1}}{b^{2}}} \\
\left.+\sqrt{\pi} \sqrt{F_{L}} \sqrt{T_{1}}\left(\frac{\sqrt{F_{L}} \sqrt{T_{1}}}{a}\right)\right) \\
\left.J_{2}\left(a, b, T_{1}, T_{2}\right)=\int_{a}^{b} r^{-2} e^{-F_{l} r^{-2} T_{1}} d r f\left(\frac{\sqrt{F_{L}} \sqrt{T_{1}}}{b}\right)\right) ; \\
\sqrt{\pi}\left(\operatorname{Erf}\left(\frac{\sqrt{F_{L}} \sqrt{T_{1}}}{a}\right)-E r f\left(\frac{\sqrt{F_{L}} \sqrt{T_{1}}}{b}\right)\right) \\
=\frac{2 \sqrt{F_{L}} \sqrt{T_{1}}}{b}
\end{array}
$$$$
\widetilde{Y}=\frac{\left|\frac{T_{1}^{-\frac{1}{2}}}{2}\right|\left|\frac{T_{2}^{-\frac{1}{2}}}{2}\right|}{Q_{1} Q_{2}} \sum_{k=1}^{\infty} \sum_{L=1}^{\infty} J\left(a, b, T_{1}, T_{2}\right)
$$

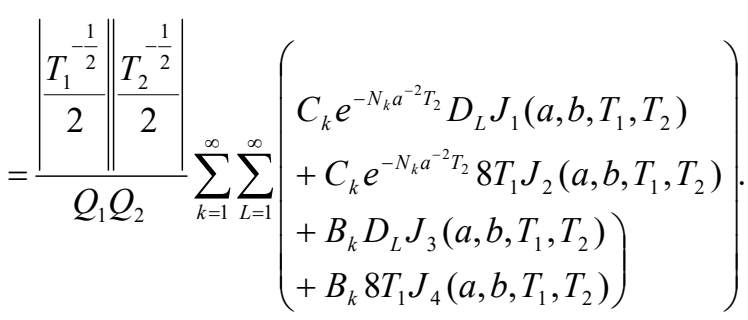

Similarly, we can find

$$
Y=\int_{0}^{\infty} F_{\bar{R}(T)}\left(\bar{r} ; T_{2}\right) \cdot f_{\bar{R}(T)}\left(\bar{r} ; T_{1}\right) d \bar{r} .
$$




\subsection{Order statistics}

For the European minimum (or maximum) options, Goldman et al. [16] defined and derived the closed form pricing formula. The exact distribution of the maximum and the minimum of the prices-path had been available among the established results in the field of mathematics (Probability Theory). There are several studies in the literature including Bergman [17], Kemna and Vorst [18], Kunitomo and Takahashi [19] and Tumbull and Wakeman [20], they determined the probability distribution of the geometric average of the prices when the underlying asset price follows the log-normal distribution, and the closed form for the option prices were obtained. However, the closed form pricing formula for the arithmetic average options do not seem to be derived yet except for a special case in Bergman [17]. The approximated pricing formula and the algorithms for them are quite well studied. The difficulty seems to be in deriving the exact distribution function of the average price. This make the order statistics are among the most fundamental tools in nonparametric statistics and inference. In this part, we discuss some properties of order statistics for TDWR.

Let $R_{1: n} \leq R_{2: n} \leq \ldots \leq R_{n: n}$ denote the order statistics of a random sample $R_{1}, R_{2}, \ldots, R_{n}$ from the TDWR. Then the p.d.f. of the $\mathrm{p}^{\text {th }}$ order $\quad$ statistic $\quad R_{p: n} \quad$ is,

$$
\begin{aligned}
g_{R(T)(p: n)} & =\frac{n !\left(G_{R(T)}(r)\right)^{p-1}\left(1-G_{R(T)}(r)\right)^{n-p} g_{R(T)}(r)}{(p-1) !(n-p) !}, \\
& =\frac{n !}{(p-1) !(n-p) !}
\end{aligned}
$$
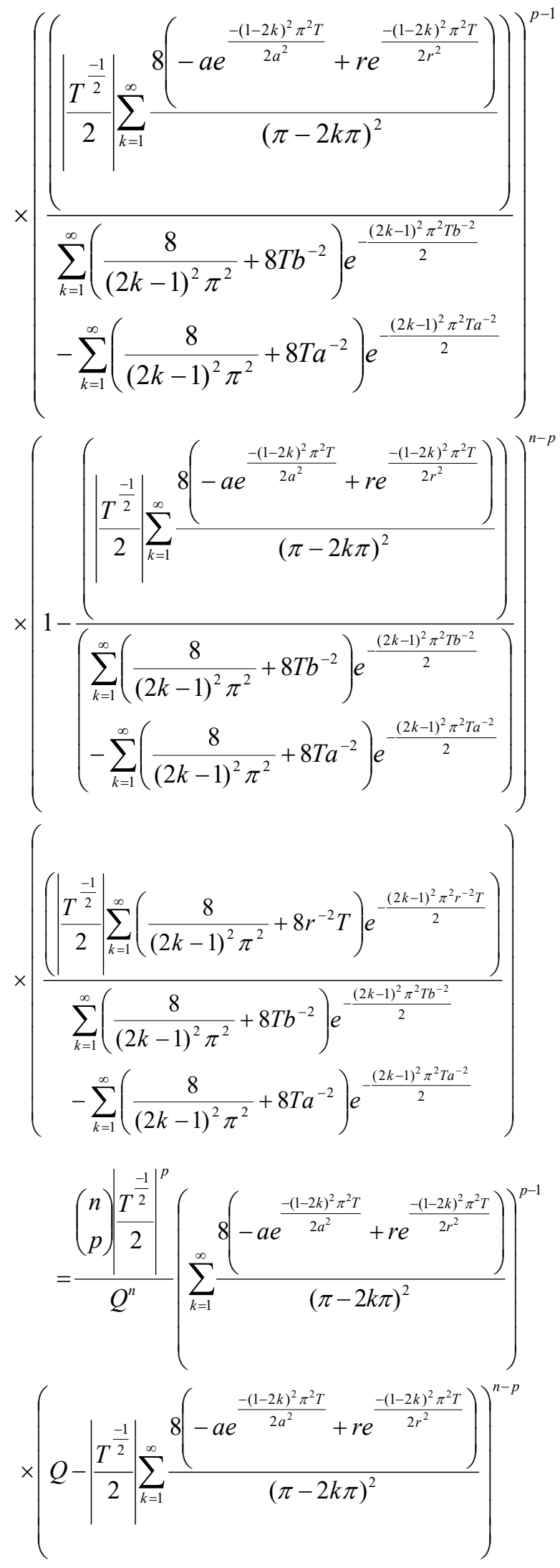


$$
\times\left(\sum_{k=1}^{\infty}\left(\frac{8}{(2 k-1)^{2} \pi^{2}}+8 r^{-2} T\right) e^{-\frac{(2 k-1)^{2} \pi^{2} r^{-2} T}{2}}\right),
$$

where,

$$
\begin{aligned}
Q= & \sum_{k=1}^{\infty}\left(\frac{8}{(2 k-1)^{2} \pi^{2}}+8 T b^{-2}\right) e^{-\frac{(2 k-1)^{2} \pi^{2} T b^{-2}}{2}} \\
& -\sum_{k=1}^{\infty}\left(\frac{8}{(2 k-1)^{2} \pi^{2}}+8 T a^{-2}\right) e^{-\frac{(2 k-1)^{2} \pi^{2} T a^{-2}}{2}}
\end{aligned}
$$

Also, the distribution function of $R_{p: n}$ is,

$$
\begin{aligned}
& G_{R(T)(p: n)}(r)=\sum_{i=p}^{n}\left(\begin{array}{l}
n \\
i
\end{array}\right)\left(G_{R(T)}(r)\right)^{i}\left(1-G_{R(T)}(r)\right)^{n-i} \\
& =\sum_{i=p}^{n}\left(\begin{array}{l}
n \\
i
\end{array}\right)\left(\frac{\left(\mid \frac{T^{\frac{-1}{2}}}{2} \sum_{k=1}^{\infty} \frac{8\left(-a e^{\frac{-(1-2 k)^{2} \pi^{2} T}{2 a^{2}}}+r e^{\frac{-(1-2 k)^{2} \pi^{2} T}{2 r^{2}}}\right)}{(\pi-2 k \pi)^{2}}\right)}{\left(\begin{array}{l}
\sum_{k=1}^{\infty}\left(\frac{8}{(2 k-1)^{2} \pi^{2}}+8 T b^{-2}\right) e^{-\frac{(2 k-1)^{2} \pi^{2} T b^{-2}}{2}} \\
-\sum_{k=1}^{\infty}\left(\frac{8}{(2 k-1)^{2} \pi^{2}}+8 T a^{-2}\right) e^{-\frac{(2 k-1)^{2} \pi^{2} T a^{-2}}{2}}
\end{array}\right)}\right) \\
& \left.\begin{array}{c}
\times\left(\mid \frac{\left.T^{\frac{-1}{2}} \mid \sum_{k=1}^{\infty} \frac{8\left(-a e^{\frac{-(1-2 k)^{2} \pi^{2} T}{2 a^{2}}}+r e^{\frac{-(1-2 k)^{2} \pi^{2} T}{2 r^{2}}}\right)}{(\pi-2 k \pi)^{2}}\right)}{\sum_{k=1}^{\infty}\left(\frac{8}{(2 k-1)^{2} \pi^{2}}+8 T b^{-2}\right) e^{-\frac{(2 k-1)^{2} \pi^{2} T b^{-2}}{2}}}\right) \\
-\sum_{k=1}^{\infty}\left(\frac{8}{(2 k-1)^{2} \pi^{2}}+8 T a^{-2}\right) e^{-\frac{(2 k-1)^{2} \pi^{2} T a^{-2}}{2}}
\end{array}\right)
\end{aligned}
$$$$
=\sum_{i=p}^{n} \frac{\left(\begin{array}{c}
n \\
i
\end{array}\right) \mid \frac{T^{\frac{-1}{2}}}{2}}{Q^{n}} \mid\left(\sum_{k=1}^{\infty} \frac{8\left(-a e^{\frac{-(1-2 k)^{2} \pi^{2} T}{2 a^{2}}}+r e^{\frac{-(1-2 k)^{2} \pi^{2} T}{2 r^{2}}}\right)}{(\pi-2 k \pi)^{2}}\right)^{i}
$$

$$
\left.Q-\left|\frac{T^{\frac{-1}{2}}}{2}\right| \sum_{k=1}^{\infty} \frac{8\left(-a e^{\frac{-(1-2 k)^{2} \pi^{2} T}{2 a^{2}}}+r e^{\frac{-(1-2 k)^{2} \pi^{2} T}{2 r^{2}}}\right)}{(\pi-2 k \pi)^{2}}\right)^{n-i}
$$

In addition, the $\mathrm{q}^{\text {th }}$ moment of the $\mathrm{p}^{\text {th }}$ order statistic $R_{p: n}$ is,

$$
\begin{array}{r}
E\left(R_{k: n}^{q}\right)=q \sum_{j=n-k+1}^{n}(-1)^{j-n+k-1}\left(\begin{array}{c}
j-1 \\
n-k
\end{array}\right)\left(\begin{array}{c}
n \\
j
\end{array}\right) \\
\times \int_{a}^{b} r^{q-1}\left(1-F_{R(T)}(r)\right)^{j} d r
\end{array}
$$

Let,

$$
\begin{aligned}
& \aleph_{j}=\int_{a}^{b} r^{q-1}\left(1-F_{R(T)}(r)\right)^{j} d r ; \\
& Z_{k}=\frac{-8 a}{(\pi-2 k \pi)^{2}} ; \text { and } \\
& G_{K}=\frac{(1-2 k)^{2} \pi^{2} T}{2} .
\end{aligned}
$$

Then,

$\aleph_{j}=\int_{a}^{b} r^{q-1}\left(1-\left|\frac{T^{-\frac{1}{2}}}{2}\right| \sum_{k=1}^{\infty}\left(Z_{k} e^{-G_{k} a^{-2}}+8 r e^{-G_{k} r^{-2}}\right)\right)^{j} d r$.

Also, let $U=\left|\frac{T^{-\frac{1}{2}}}{2}\right| \sum_{k=1}^{\infty}\left(Z_{k} e^{-G_{k} a^{-2}}+8 r e^{-G_{k} r^{-2}}\right)$.

From Binomial uniforms theorem we get:

$$
(1-U)^{j}=\sum_{j=0}^{p}\left(\begin{array}{l}
p \\
j
\end{array}\right)(-1)^{j} U^{j} \text {. }
$$

Thus, 


$$
\begin{aligned}
& \aleph_{j}=\int_{a}^{b} r^{q-1}(1-U)^{j} d r \\
&= \sum_{j=0}^{p}\left(\begin{array}{l}
p \\
j
\end{array}\right)(-1)^{j} \int_{a}^{b} U^{j} r^{q-1} d r \\
&=\sum_{j=0}^{p}\left(\begin{array}{c}
p \\
j
\end{array}\right)(-1)^{j} \\
&\left.\quad \times\left(\mid \frac{T^{-\frac{1}{2}}}{2}\right)\right)^{j} \int_{a}^{b} r^{q-1}\left(\sum_{k=1}^{\infty}\left(\begin{array}{l}
Z_{k} e^{-G_{k} a^{-2}} \\
+8 r e^{-G_{k} r^{-2}}
\end{array}\right)\right)^{j} d r
\end{aligned}
$$

At $\quad j=0 \quad$ we get, $\quad \aleph_{0}=\left(\begin{array}{c}p \\ o\end{array}\right)_{a}^{b} r^{q-1} d r$

$=\frac{b^{q}-a^{q}}{q} . \quad$ And, at $j=1 \quad$ we have,

$\aleph_{1}=\left(\begin{array}{c}p \\ 1\end{array}\right)(-1) \frac{T^{-\frac{1}{2}}}{2} \mid \int_{a}^{b} \sum_{k=1}^{\infty}\left(\begin{array}{l}Z_{k} e^{-G_{k} a^{-2}} \\ +8 r e^{-G_{k} r^{-2}}\end{array}\right) r^{q-1} d r$ $=\left(\begin{array}{l}p \\ 1\end{array}\right)(-1)\left|\frac{T^{-\frac{1}{2}}}{2}\right| \sum_{k=1}^{\infty} \int_{a}^{b}\left(\begin{array}{l}Z_{k} e^{-G_{k} a^{-2}} \\ +8 r e^{-G_{k} r^{-2}}\end{array}\right) r^{q-1} d r$ $=\left(\begin{array}{l}p \\ 1\end{array}\right)(-1)\left|\frac{T^{-\frac{1}{2}}}{2}\right|$

$$
\sum_{k=1}^{\infty} \frac{\left(+\frac{G_{k}}{a^{2}}\left(-4 e^{-\frac{G_{k}}{a^{2}}} q\left(\begin{array}{l}
\left.a^{1+q} E i\left(\frac{3+q}{2}, \frac{G_{k}}{a^{2}}\right)\right) \\
\left.-b^{1+q} E i\left(\frac{3+q}{2}, \frac{G_{k}}{b^{2}}\right)\right)
\end{array}\right)\right.\right.}{q\left(-a^{q}+b^{q}\right) Z_{k}}
$$

where Ei gives the exponential integral function. Also, at $j=2$ we obtain,

$\aleph_{2}=\left(\begin{array}{l}p \\ 2\end{array}\right)\left(\left|\frac{T^{-\frac{1}{2}}}{2}\right|\right)^{2} \int_{a}^{b}\left(\sum_{k=1}^{\infty}\left(\begin{array}{l}Z_{k} e^{-G_{k} a^{-2}} \\ +8 r e^{-G_{k} r^{-2}}\end{array}\right)\right)^{2} r^{q-1} d r$.

Since,

$$
\left(\sum_{k=1}^{\infty}\left(\begin{array}{l}
Z_{k} e^{-G_{k} a^{-2}} \\
+8 r e^{-G_{k} r^{-2}}
\end{array}\right)\right)^{2}=\sum_{k=1}^{\infty} \sum_{n=1}^{\infty}\left(\begin{array}{l}
Z_{k} e^{-G_{k} a^{-2}} \\
+8 r e^{-G_{k} r^{-2}}
\end{array}\right)\left(\begin{array}{l}
Z_{n} e^{-G_{n} a^{-2}} \\
+8 r e^{-G_{n} r^{-2}}
\end{array}\right)
$$

$$
=\sum_{k=1}^{\infty} \sum_{n=1}^{\infty}\left(\begin{array}{l}
Z_{k} Z_{n} e^{-\left(G_{k}+G_{n}\right) a^{-2}}+8 r Z_{k} e^{-G_{k} a^{-2}-G_{n} r^{-2}} \\
+8 r Z_{n} e^{-G_{k} r^{-2}-G_{n} a^{-2}}+64 r^{2} e^{-G_{k} r^{-2}-G_{n} r^{-2}}
\end{array}\right) .
$$

Thus,

$$
\begin{aligned}
& \left.\aleph_{2}=\left(\begin{array}{l}
p \\
2
\end{array}\right)\left(\mid \frac{T^{-\frac{1}{2}}}{2}\right)\right)^{2} \sum_{k=1}^{\infty} \sum_{n=1}^{\infty} \int_{a}^{b}\left(\begin{array}{l}
Z_{k} Z_{n} e^{-\left(G_{k}+G_{n}\right) a^{-2}} \\
+8 r Z_{k} e^{-G_{k} a^{-2}-G_{n} r^{-2}} \\
+8 r Z_{n} e^{-G_{k} r^{-2}-G_{n} a^{-2}} \\
+64 r^{2} e^{-G_{k} r^{-2}-G_{n} r^{-2}}
\end{array}\right) r^{q-1} d r \\
& =\left(\begin{array}{l}
p \\
2
\end{array}\right)\left(\mid \begin{array}{l}
Z_{k} Z_{n} \int_{a}^{b} e^{-\left(G_{k}+G_{n}\right) a^{-2}} r^{q-1} d r \\
2 \\
+8 Z_{K} \int_{a}^{b} r^{q} e^{-G_{k} a^{-2}-G_{n} r^{-2}} d r \\
+8 Z_{n} \int_{a}^{b} r^{q} e^{-G_{k} r^{-2}-G_{n} a^{-2}} \\
d r+64 \int_{a}^{b} r^{q+1} e^{-\left(G_{k}+G_{n}\right) r^{-2}} d r
\end{array}\right),
\end{aligned}
$$

where,

$$
\begin{aligned}
& \int_{a}^{b} e^{-\left(G_{k}+G_{n}\right) a^{-2}} r^{q-1} d r=e^{-\left(G_{k}+G_{n}\right) a^{-2}} \frac{b^{q}-a^{q}}{q} ; \\
& \int_{a}^{b} r^{q} e^{-G_{k} a^{-2}-G_{n} r^{-2}} d r=\frac{1}{2} e^{-\frac{G_{k}}{a^{2}}}\left(\begin{array}{l}
-a^{q+1} E i\left(\frac{3+q}{2}, \frac{G_{n}}{a^{2}}\right) \\
+b^{q+1} E i\left(\frac{3+q}{2}, \frac{G_{n}}{b^{2}}\right)
\end{array}\right)
\end{aligned}
$$

and

$$
\begin{aligned}
\int_{a}^{b} r^{q+1} e^{-\left(G_{k}+G_{n}\right) r^{-2}} d r=\frac{1}{2}\left(G_{k}+G_{n}\right) \\
\times\left(\begin{array}{l}
-a^{q} \Gamma\left(-1-\frac{q}{2}, \frac{G_{k}+G_{n}}{a^{2}}\right)\left(\frac{a^{2}}{G_{k}+G_{n}}\right)^{-\frac{Q}{2}} \\
+b^{q} \Gamma\left(-1-\frac{q}{2}, \frac{G_{k}+G_{n}}{b^{2}}\right)\left(\frac{b^{2}}{G_{k}+G_{n}}\right)^{-\frac{Q}{2}}
\end{array}\right)
\end{aligned}
$$

By the same method we can obtain

$$
\aleph_{j}=\int_{a}^{b} r^{q-1}(1-U)^{j} d r
$$

\subsection{Bonferroni curve, Lorenz curve and Gini's index}

Recently, studies of the stock price has gained a lot of importance. Some important measures in this studies are the Lorenz curve and Gini's 
index. Lorenzcurve and the associated Gini index are undoubtedly the most popular indices of income in equality. Giorgi and Mondani [9] and Giorgi [10] shown that Bonferroni curve is such a measure, which has the advantage of being represented graphically in the unit square and can also be related to the Lorenz Curve and Gini ratio. Giorgi and Crescenzi [11] presented that these measures have some applications in reliability and life testing as well.

Since $R$ be a non negative random variable with cumulative distribution function (4) which is smooth (i.e., continuous and has derivatives of all orders). However, the first moment of $R$ about zero is finite, exists and non zero as in (20). The Lorenz curve is useful in business modeling: e.g., in consumer finance, to measure the actual percentage of delinquencies attributable to the percentage of people with worst risk scores. Lorenz curve can be obtained by using the equation,

$$
\begin{aligned}
& L\left(g_{R(T)}(r)\right)=\frac{\int_{a}^{r} r g_{R(T)}(r) d r}{\int_{a}^{b} r g_{R(T)}(r) d r} \\
& =\frac{\sum_{k=1}^{\infty}\left[\begin{array}{c}
-4 T\left(\begin{array}{l}
\Gamma\left[0, \frac{(1-2 k)^{2} \pi^{2} T}{2 a^{2}}\right] \\
-\Gamma\left[0, \frac{(1-2 k)^{2} \pi^{2} T}{2 r^{2}}\right]
\end{array}\right] \\
\left.+\frac{A}{(\pi-2 k \pi)^{2}}\right]
\end{array},\right.}{\sum_{k=1}^{\infty}\left[\begin{array}{c}
\Gamma\left[0, \frac{(1-2 k)^{2} \pi^{2} T}{2 a^{2}}\right] \\
\left.-4 T\left[0, \frac{(1-2 k)^{2} \pi^{2} T}{2 b^{2}}\right]\right) \\
+\frac{C}{(\pi-2 k \pi)^{2}}
\end{array}\right]}
\end{aligned}
$$

where,

$$
\begin{gathered}
A=2\left[\begin{array}{l}
-2 a^{2} e^{-\frac{(1-2 k)^{2} \pi^{2} T}{2 a^{2}}}+2 r^{2} e^{-\frac{(1-2 k)^{2} \pi^{2} T}{2 r^{2}}} \\
+(1-2 k)^{2} \pi^{2} T \cdot \Gamma\left[0, \frac{(1-2 k)^{2} \pi^{2} T}{2 a^{2}}\right] \\
-(1-2 k)^{2} \pi^{2} T \cdot \Gamma\left[0, \frac{(1-2 k)^{2} \pi^{2} T}{2 r^{2}}\right]
\end{array}\right], \\
C=2\left[\begin{array}{l}
-2 a^{2} e^{-\frac{(1-2 k)^{2} \pi^{2} T}{2 a^{2}}}+2 b^{2} e^{-\frac{(1-2 k)^{2} \pi^{2} T}{2 b^{2}}} \\
+(1-2 k)^{2} \pi^{2} T \cdot \Gamma\left[0, \frac{(1-2 k)^{2} \pi^{2} T}{2 a^{2}}\right] \\
-(1-2 k)^{2} \pi^{2} T \cdot \Gamma\left[0, \frac{(1-2 k)^{2} \pi^{2} T}{2 b^{2}}\right]
\end{array}\right] .
\end{gathered}
$$

The Gini index which is defined as a ratio of the areas on the Lorenez curve is given by:

$$
G=1-\frac{2}{Z} \sum_{k=1}^{\infty}\left[\begin{array}{l}
-4 T(b-a) \Gamma\left[0, \frac{(1-2 k)^{2} \pi^{2} T}{2 a^{2}}\right] \\
+4 T(W+\chi)+ \\
{\left[\begin{array}{l}
\Lambda+4 Y+S- \\
\left(2 k(1-2 k)^{2}(1-2 k)^{2} \pi^{2} T(W+\chi)\right)
\end{array}\right]} \\
\frac{(\pi-2 k \pi)^{2}}{2}
\end{array}\right],
$$

where,

$$
\begin{aligned}
& W=-\sqrt{2}(1-2 k) \pi^{\frac{3}{2}} \sqrt{T} \operatorname{Erf}\left[\frac{(-1+2 k) \pi \sqrt{T}}{\sqrt{2} a}\right] \\
& +\sqrt{2}(1-2 k) \pi^{\frac{3}{2}} \sqrt{T} \operatorname{Erf}\left[\frac{(-1+2 k) \pi \sqrt{T}}{\sqrt{2} b}\right] \text {; } \\
& \chi=-a\left[-2 e^{-\frac{(1-2 k)^{2} \pi^{2} T}{2 a^{2}}}+\Gamma\left[0, \frac{(1-2 k)^{2} \pi^{2} T}{2 a^{2}}\right]\right] \\
& +b\left[-2 e^{-\frac{(1-2 k)^{2} \pi^{2} T}{2 b^{2}}}+\Gamma\left[0, \frac{(1-2 k)^{2} \pi^{2} T}{2 b^{2}}\right]\right] ; \\
& Y=-\frac{1}{6} e^{-\frac{\left(1+4 k^{2}\right) \pi^{2} T}{2 a^{2}}}\left[\begin{array}{l}
2 a e^{\frac{2 k \pi^{2} T}{a^{2}}}\left(a^{2}-(1-2 k)^{2} \pi^{2} T\right) \\
-\left[\begin{array}{l}
\sqrt{2} e^{-\frac{\left(1+4 k^{2}\right) \pi^{2} T}{2 a^{2}}}(-1+2 k)^{3} \pi^{\frac{7}{2}} \\
\times T^{\frac{3}{2}} \operatorname{Erf}\left[\frac{(-1+2 k) \pi \sqrt{T}}{\sqrt{2} a}\right]
\end{array}\right]
\end{array}\right]
\end{aligned}
$$




$$
\begin{aligned}
& +\frac{1}{6} e^{-\frac{\left(1+4 k^{2}\right) \pi^{2} T}{2 b^{2}}}\left[\begin{array}{l}
2 b e^{\frac{2 k \pi^{2} T}{b^{2}}}\left(b^{2}-(1-2 k)^{2} \pi^{2} T\right) \\
-\left[\begin{array}{c}
\sqrt{2} e^{-\frac{\left(1+4 k^{2}\right) \pi^{2} T}{2 b^{2}}}(-1+2 k)^{3} \pi^{\frac{7}{2}} \\
\times T^{\frac{3}{2}} \operatorname{Erf}\left[\frac{(-1+2 k) \pi \sqrt{T}}{\sqrt{2} b}\right]
\end{array}\right]
\end{array}\right] ; \\
& Z=\sum_{k=1}^{\infty}\left[\begin{array}{l}
-4 T\left[\begin{array}{l}
\Gamma\left[0, \frac{(1-2 k)^{2} \pi^{2} T}{2 a^{2}}\right] \\
-\Gamma\left[0, \frac{(1-2 k)^{2} \pi^{2} T}{2 b^{2}}\right]
\end{array}\right) \\
+\frac{C}{(\pi-2 k \pi)^{2}}
\end{array}\right] \text {; }
\end{aligned}
$$

$C$ is given above.

$$
S=2(1-2 k)^{2}(b-a) \pi^{2} T \cdot \Gamma\left[0, \frac{(1-2 k)^{2} \pi^{2} T}{2 a^{2}}\right]
$$

and $\Lambda=-4 a^{2}(b-a) e^{-(1-2 k)^{2} \pi^{2} T}$. Also, the

Bonferroni curve is given by:

$$
B_{g}\left(g_{R(T)}(r)\right)=\frac{L\left(g_{R(T)}(r)\right)}{G_{R(T)}(r)},
$$

where $L\left(g_{R(T)}(r)\right)$ is given by (22) and from

(4) we get $G_{R(T)}(r)$.

\section{Application}

The oscillation between the fall and rise of the stock price within a time period $T$ can be expressed by a Wiener process. The difference between the highest and the lowest value of the stock price it called the range $R$ of the Wiener process. When the selling price becomes equal to the cost price then $R=0$ and when the share price up to the upper limit barrier (the upper limit that the stock price has already been reached and reversed to decline) then $R=\infty$. In the upper limit barrier case, the analysts believe that the stock price became expensive and there is no rush to buy it. In this case, sudden drop in the market index may occur while the stock did not reach the point of sale. To avoid a sudden drop in the share price sale we should study the behavior of $R$ by studying some its statistical properties as in Withers and Nadarajah [2]. To ensure that no loss, we should put an upper limit barrier (to avoid sudden drop) and lower limit barrier greater than 0 (a guarantee of a gain even if few). Thus, we use (3) and (4) to get these statistical properties of the bounded range.

In [2], Withers and Nadarajah supposed that $x=r T^{-\frac{1}{2}} / 2$ where the values of $x$ are given. Here, we let the truncated values of $x_{\kappa}, \kappa=1,2, \ldots, 5$ for the corresponding time periods are $T_{v}, v=1,2, \ldots, 5$, then we get the values of the lower limit barrier $a$ and the upper limit barrier $b$. Also, we obtain the values of $R$, the probability density function and cumulative distribution function of $R$ are given in Table 1.

Table 1: The probability density function, cumulative distribu of $R$.

\begin{tabular}{cccc}
\hline$T_{v}$ & $a \leq r \leq b$ & $r$ & $g_{R(T)}(r)$ \\
\hline & & 14.14 & 0.359021 \\
& & 15.2 & 0.383331 \\
50 & $14.14 \leq r \leq 19.799$ & 15.5 & 0.3899 \\
& & 15.556 & 0.389935
\end{tabular}


The most important information for the company that builds its decision in order to choose the right time to sell the stock when $a \leq R \leq b$ is to know the mean value of $R$. From (17) we find that the mean value of $R$ is depend on the values of $a, b$ and $T$ as follows:

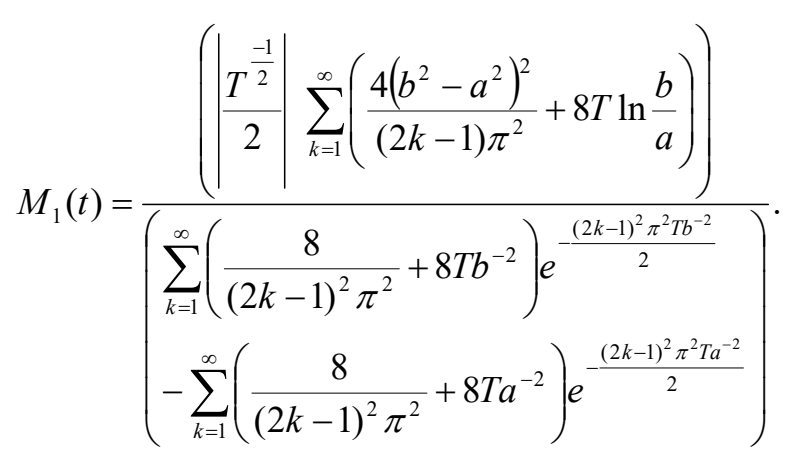

Also, the mean values of $R$ for the corresponding time periods $T_{v}, v=1,2, \ldots, 5$ are given in Table 1 .

By using mathematica 7 , we found that $\sum_{k=1}^{1000000} \frac{8}{(2 k-1) \pi^{2}} \approx 1$. Thus, in (3), (4) and $M_{1}(t)$ we get the values of the probability density function, cumulative distribution function and the mean values of $R$ as in Table 1.

\section{Table 1: Continued}

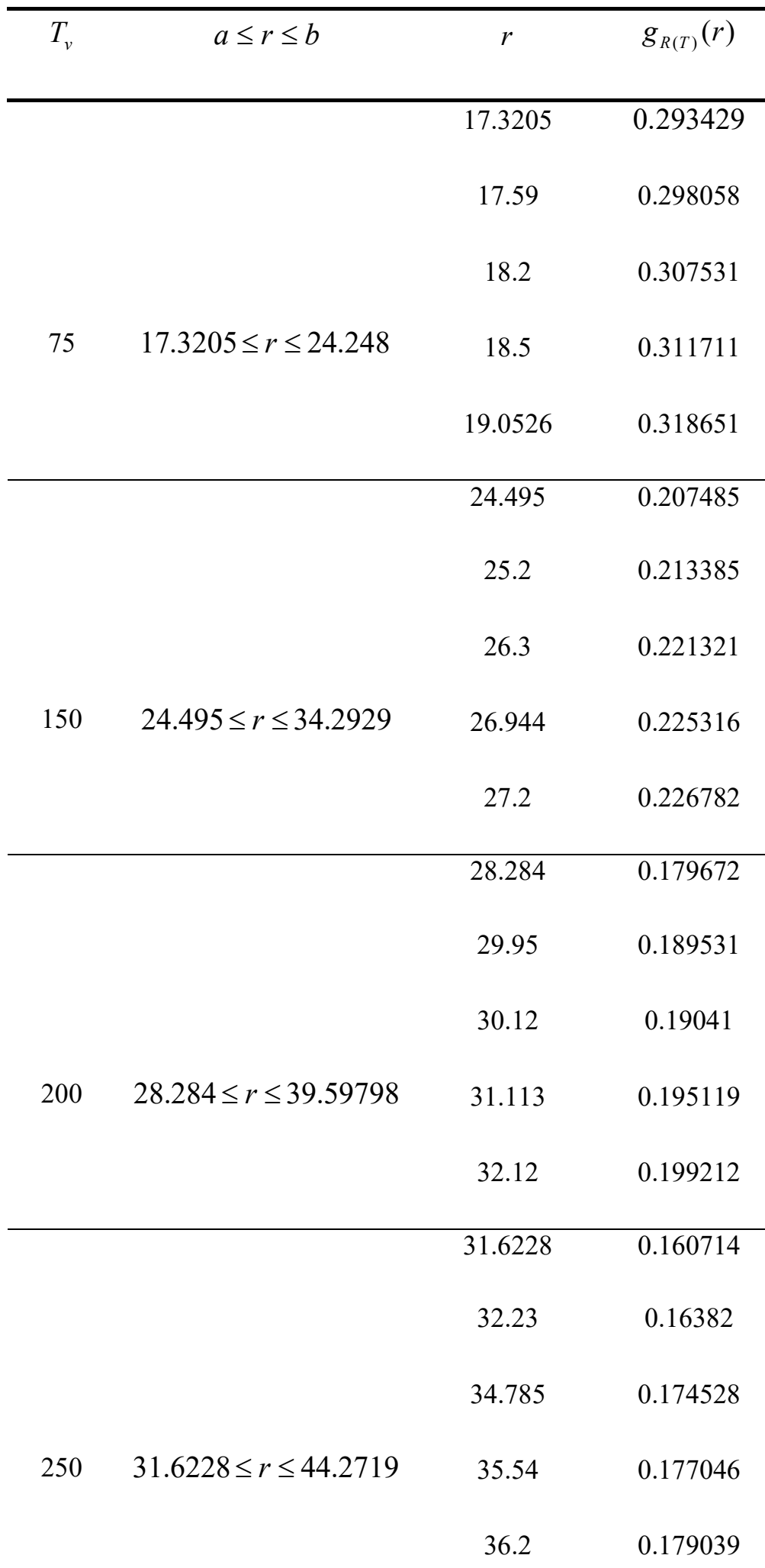




\section{Concluding remarks}

In this paper we introduced a truncated distribution for the range of a Wiener process. This distribution is the best for the stock price in a limited range. We provided a mathematical treatment to find some statistical properties including reliability properties, moments, stress-strength parameter, order statistics, Bonferroni curve, Lorenz curve and Gini's index. A real data set is analyzed to clarify the effectiveness of this distribution. We hope that this distribution may attract a wide applications in lifetime modeling.

In future research one can introduce a new type of middle and random truncation for the range of a Wiener process.

\section{Acknowledgements}

The authors are very grateful to the Editor in Chief and the anonymous referees for their careful reading of the paper, and for suggestions which helped the author to improve it.

\section{Appendix A}

To get a double truncated density function of

TDRW, we put $x=r T^{-\frac{1}{2}} / 2, \quad \bar{a}=\frac{a T^{-\frac{1}{2}}}{2}$, $\bar{b}=\frac{b T^{-\frac{1}{2}}}{2}$, and $\omega_{k}=(2 k-1)^{2} \pi^{2} / 8$, then (1) (the probability density function of the range) become:

$$
\phi_{T}(x)=\sum_{k=1}^{\infty}\left[\begin{array}{c}
\left(-4 x^{-3} \exp \left(-\omega_{k} x^{-2}\right)\right) \\
+\left(2 \omega_{k} x^{-3} \exp \left(-\omega_{k} x^{-2}\right)\right) \\
\times\left(\omega_{k}^{-1}+2 x^{-2}\right)
\end{array}\right] .
$$


And, its cumulative distribution function is given by:

$\Phi_{T}(x)=\sum_{k=1}^{\infty}\left(\omega_{k}^{-1}+2 \bar{a}^{-2}\right) \exp \left(-\omega_{k} x^{-2}\right)$

Consequently,

$\Phi_{T}(\bar{a})=\sum_{k=1}^{\infty}\left(\omega_{k}^{-1}+2 \bar{a}^{-2}\right) \exp \left(-\omega_{k} \bar{a}^{-2}\right)$ and

$\Phi_{T}(b)=\sum_{k=1}^{\infty}\left(\omega_{k}^{-1}+2 \bar{b}^{-2}\right) \exp \left(-\omega_{k} \bar{b}^{-2}\right)$.If

$\bar{a} \leq x \leq \bar{b}$ then the double truncated density function can get from the equation:

$$
\begin{aligned}
\bar{\phi}_{T}(x) & =\frac{\phi_{T}(x)}{\Phi_{T}(\bar{b})-\Phi_{T}(\bar{a})} \\
& =\frac{\sum_{k=1}^{\infty}\left[\begin{array}{c}
\left(-4 x^{-3} \exp \left(-\omega_{k} x^{-2}\right)\right) \\
+\left(2 \omega_{k} x^{-3} \exp \left(-\omega_{k} x^{-2}\right)\right) \\
\times\left(\omega_{k}^{-1}+2 x^{-2}\right)
\end{array}\right]}{\left[\begin{array}{c}
\sum_{k=1}^{\infty}\left(\omega_{k}^{-1}+2 \bar{b}^{-2}\right) \exp \left(-\omega_{k} \bar{b}^{-2}\right) \\
-\sum_{k=1}^{\infty}\left(\omega_{k}^{-1}+2 \bar{a}^{-2}\right) \exp \left(-\omega_{k} \bar{a}^{-2}\right)
\end{array}\right]}
\end{aligned}
$$

Now by substituent again with $x=\frac{r T^{-\frac{1}{2}}}{2}, \quad \bar{a}=\frac{a T^{-\frac{1}{2}}}{2}, \quad \bar{b}=\frac{b T^{-\frac{1}{2}}}{2}$, and $\omega_{k}=(2 k-1)^{2} \pi^{2} / 8$ then the density function of TDWR is given by:

$$
\begin{aligned}
& \Gamma_{R(T)}(r)=\frac{f_{\bar{R}(T)}(r)}{F_{\bar{R}(T)}(\bar{b})-F_{\bar{R}(T)}(\bar{a})} \\
& =\frac{\left[\left|\frac{T^{-\frac{1}{2}}}{2}\right| \sum_{k=1}^{\infty}\left(\frac{8}{(2 k-1)^{2} \pi^{2}}+8 r^{-2} T\right) e^{-\frac{(2 k-1)^{2} \pi^{2} r^{-2} T}{2}}\right]}{\left[\sum_{k=1}^{\infty}\left(\frac{8}{(2 k-1)^{2} \pi^{2}}+2 T b^{-2}\right) e^{-\frac{(2 k-1)^{2} \pi^{2} T b^{-2}}{8}}\right]} . \\
& \left.-\sum_{k=1}^{\infty}\left(\frac{8}{(2 k-1)^{2} \pi^{2}}+2 T a^{-2}\right) e^{-\frac{(2 k-1)^{2} \pi^{2} T a^{-2}}{8}}\right]
\end{aligned}
$$

Using integration by parts one can shows that $\int_{a}^{b} \Gamma_{R(T)}(r) d r=1$.

\section{References}

[1] W. Feller, The asymptotic distribution of the range of sums of independent random variables, Ann. Math. Stat., 22 (1951) 427-432.

[2] C. Withers and S. Nadarajah, The distribution and quantiles of the range of a Wiener process, Appl. Math. and Compu., 232 (2014) 766-770.

[3] M. Ali and S. Nadarajah, A truncated Pareto distribution, Compu. Commun.,30 (2006)1-4.

[4] S. Nadarajah, Some truncated distributions, Acta Appl. Math., 106(2009) 105-123.

[5] L. Zaninetti and M. Ferraro, On the truncated Pareto distribution with applications, Cent. Eur. J. Phys., 6(1)(2008) 1-6.

[6] J.D. Church and B. Harris, The estimation of reliability from stress strength relationships, Technometrics 12 (1970) 49-54.

[7] J. Pender, The truncated normal distribution: Applications to queues with impatient customers, Operations Research Letters 43 (2015) 40-45.

[8] S. Chattopadhyay, C.A. Murthy and S. K. Pal, Fitting truncated geometric distributions in large scale real world networks, Theoretical Computer Science 551 (2014) 22-38.

[9] G.M. Giorgi and R. Mondani, Sampling distribution of Bonferroni inequality index from exponential population, Sankhya B 57 (1995), 10-18.

[10] G.M. Giorgi, Concentration Index, Bonferroni. Encyclopedia of Statistical Sciences, Wiley, New York, 2(1998), 141-146. 
[11] G.M. Giorgi and M. Crescenzi, A look at the Bonferroni inequality measure in a reliability framework, StatisticaLXL 4 (2001), 571-583.

[12] L. Zaninetti, A right and left truncated gamma distribution with application to the stars. Advanced Studies in Theoretical Physics, 23 (2014), 1139-1147.

[13] S.H. Alkarrni, A class of truncated binomial lifetime distributions, Open Journal of Statistics 3(2013), 305 -313.

[14] M.M. Mohie El-Din, A.A. Teamah, A. ElBar and A. Salem, Random sum of mid truncated Lindley distribution, Journal of Advanced Research in Statistics and Probability, 2(1) (2010), 27-36.

[15] A.A. Teamah, A. El-Bar and A. Salem, Random sum of truncated and random truncated Lindley distribution, the Int. Journal of Applied Mathematics, 23 (2010), 961-971.

[16] M. Goldman, H.B. Sosin and M.A. Gatto, Path Dependent Options; 'Buy at the low,
Sell at the High, Journal of Finance, 34 (1979) 1111-1127.

[17] Z. Bergman, Pricing Path Contingent Claims," Research in Finance, 5 (1985) 229-241.

[18] A. Kemna and A.Vorst, A Pricing Method for Options Based on Average Asset Values, Journal of Banking and Finance, 14 (1990) 113-129.

[19] N. Kunitomo and A. Takahashi, Pricing Method for Average Options, Finance Kenkyu, 14 (1992) 1-19. (in Japanese)

[20] M. Turnbull and L. Wakeman, A Quick Algorithm for Pricing European Average Options, Journal of Financial and Quantitative Analysis, 26 (1991) 377-389.

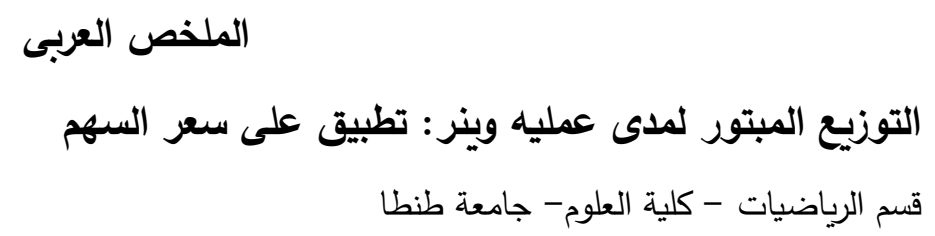

فـى هذه الورقهـ تم ايجـاد توزيع سـعر السـهم لمنتج فى فتره محدده عن طريق ايجـاد التوزيح الاحتمـالى المبتور لمدى عملية العشوائيه. تم ايجاد الخصائص الإحصائية المختلفة للتوزيع بمـا في ذلك خصائص الموثوقية، العزوم، معامل الاجهاد والقوة ،الإحصـاءات الرتيبـة، ومنحنى Bonferroni ، منحنى Lorenz ومعامـل Gini. تم اعطـاء مثنال يحتوى على مجموعـه مفترضه من البيانات لتوضيح فعالية هذا التوزيع. 